\title{
Time delay and magnification centroid due to gravitational lensing by black holes and naked singularities
}

\author{
K. S. Virbhadra* \\ Department of Mathematics, Physics and Statistics, \\ University of the Sciences in Philadelphia, Philadelphia, PA 19104 \\ C. R. Keetori \\ Department of Physics and Astronomy, Rutgers University, \\ 136 Frelinghuysen Road, Piscataway, NJ 08854
}

\begin{abstract}
We model the massive dark object at the center of the Galaxy as a Schwarzschild black hole as well as Janis-Newman-Winicour naked singularities, characterized by the mass and scalar charge parameters, and study gravitational lensing (particularly time delay, magnification centroid, and total magnification) by them. We find that the lensing features are qualitatively similar (though quantitatively different) for the Schwarzschild black holes, weakly naked, and marginally strongly naked singularities. However, the lensing characteristics of strongly naked singularities are qualitatively very different from those due the Schwarzschild black holes. The images produced by Schwarzschild black hole lenses and weakly naked and marginally strongly naked singularity lenses always have positive time delays. On the other hand, the strongly naked singularity lenses can give rise to images with positive, zero, or negative time delays. In particular, for a large angular source position the direct image (the outermost image on the same side as the source) due to strongly naked singularity lensing always has negative time delay. We also found that the scalar field decreases the time delay and increases the magnitude of magnifications of images; this result could have important implications for cosmology. As the Janis-Newman-Winicour metric also describes the exterior gravitational field of a scalar star, naked singularities as well as scalar star lenses, if these exist in nature, will serve as more efficient cosmic telescopes than regular gravitational lenses.
\end{abstract}

PACS numbers: 95.30.sf, 04.20.Dw, 04.70.Bw, 98.62.Sb

\section{INTRODUCTION}

A naked (visible) singularity is defined as a spacetime singularity which can be seen by some observer and also lies to the future of some point of the spacetime [1]. The well-known weak cosmic censorship hypothesis (WCCH) of Penrose essentially states that, generically, spacetime singularities of physically realistic gravitational collapse are hidden within black holes [1, 2]. The concept of visible singularities is objectionable to many scientists, as their existence is thought to have alarming astrophysical implications. On the other hand, a failure of the WCCH will give us the great opportunity to probe the extremely strong gravitational fields that will help in the discovery of the physical laws of quantum gravity. Despite many industrious efforts, we are still far from having a general proof (or disproof) of this hypothesis, and Penrose [1] expected that radically new mathematical techniques might be required for this purpose. As a proof or disproof of this hypothesis appears to be inordinately difficult, it may be easier to find a persuading counterexample to demonstrate that the hypothesis is not correct. Numerous diligent efforts have been put in this direction in the last four decades; however, we still do not have a single

\footnotetext{
*Email address : shwetket@yahoo.com

${ }^{\dagger}$ Email address : keeton@physics.rutgers.edu
}

convincing counterexample to the WCCH (see references in [1, 2, 3] ). In a seminal review, Penrose [1] concluded that the question of the cosmic censorship is still very much open and considered this to be possibly the most important unsolved problem in classical general relativity.

Given that we have neither a proof (or disproof) nor a convincing counterexample of the $\mathrm{WCCH}$, it is important to explore whether or not this hypothesis could be tested observationally. To this end, Virbhadra et al. [4] introduced a theoretical research project using the gravitational lensing phenomena and encouraging results came out of that. Further, Virbhadra and Ellis 5] obtained a new gravitational lens equation that allows large deflection of light and therefore it can be used to study strong gravitational field lensing. They used this lens equation to study the gravitational lensing due to light deflection close to the photon sphere of the supermassive "black hole" at the center of the Galaxy. They found that the presence of a photon sphere gives rise to a theoretically infinite sequence of highly demagnified images on both sides of the optical axis (the line joining the lens and the observer) and they termed these relativistic images. Virbhadra and Ellis 7] further extended the previous studies of Virbhadra et al. [4] in detail. They also organized the investigations by classifying naked singularities in two groups: weakly naked and strongly naked singularities. They modeled massive dark objects at the centers of a few galaxies as Schwarzschild black holes and Janis- 
Newman-Winicour weakly as well as strongly naked singularities, and studied gravitational lensing by them. The Schwarzschild black holes as well as weakly naked singularities have qualitatively similar lensing characteristics : Both have one Einstein ring and no radial critical curves. On the other hand, the strongly naked singularities have qualitatively different lensing features, i.e., they give rise to two or none Einstein rings and one radial critical curve. After publication of these results [4, 5, 6, 7], there has been a growing curiosity in black hole lensing and many interesting papers have appeared in last few years (see [8, 9, 10, 11, 12, 13, 14, 15, 16, 17, 18, 19] and references therein).

In this paper, we study the time delay, magnification centroid, and total magnification of images due to gravitational lensing by Schwarzschild black holes and JanisNewman-Winicour naked singularities. One of the most striking results in this paper is that the strongly naked singularities can give rise to images with negative time delays. We use geometrized units (i.e., $G=1, c=1$ ) throughout this paper; however, we finally compute time delays in terms of minutes. We use MATHEMATICA [20] for computations.

\section{LENS EQUATION, LIGHT DEFLECTION ANGLE, AND CLASSIFICATION OF NAKED SINGULARITIES}

In this Section, we write in brief some of the results obtained in previous papers $([4,5,6,67,21,22])$ and refine the classification of naked singularities given in [7], because these are required for computations and analysis of results in this paper.

Virbhadra and Ellis [5] derived a gravitational lens equation that permits small as well as large bending angle of light, and that is given by

$$
\tan \beta=\tan \theta-\alpha
$$

with

$$
\alpha \equiv \frac{D_{d s}}{D_{s}}[\tan \theta+\tan (\hat{\alpha}-\theta)]
$$

$D_{s}, D_{d s}$ and $D_{d}$, respectively, are the observer-source, the lens-source, and the observer-lens distances. $\hat{\alpha}$ is the light bending angle. $\theta$ and $\beta$ are, respectively, angular positions of an image and an unlensed source measured from the optical axis. (See Fig. 1 in [5].) The impact parameter $J=D_{d} \sin \theta$. For small angles, the Eq. (1) reduces to the most well-known lens equation used for studying lensing in a weak gravitational field [23].

In circularly symmetric gravitational lensing, the magnification $\mu$ of an image is

$$
\mu=\left(\frac{\sin \beta}{\sin \theta} \frac{d \beta}{d \theta}\right)^{-1}
$$

The tangential and radial magnifications are respectively expressed by

$$
\mu_{t}=\left(\frac{\sin \beta}{\sin \theta}\right)^{-1} \text { and } \quad \mu_{r}=\left(\frac{d \beta}{d \theta}\right)^{-1} .
$$

The singularities in $\mu_{t}$ and $\mu_{r}$ in lens plane give, respectively, tangential critical curves (TCCs) and radial critical curves (RCCs), and their corresponding values in the source plane are, respectively, known as the tangential caustic (TC) and radial caustics ( $\mathrm{RCs})$.

Virbhadra et al. [4] considered a general static and spherically symmetric spacetime described the line element

$$
d s^{2}=B(r) d t^{2}-A(r) d r^{2}-D(r) r^{2}\left(d \vartheta^{2}+\sin ^{2} \vartheta d \varphi^{2}\right)
$$

and calculated the deflection angle $\hat{\alpha}\left(r_{0}\right)$ and impact parameter $J\left(r_{0}\right)$ for a light ray with the closest distance of approach $r_{0}$. These are given by

$\hat{\alpha}\left(r_{0}\right)=2 \int_{r_{0}}^{\infty}\left(\frac{A(r)}{D(r)}\right)^{1 / 2}\left[\left(\frac{r}{r_{0}}\right)^{2} \frac{D(r)}{D\left(r_{0}\right)} \frac{B\left(r_{0}\right)}{B(r)}-1\right]^{-1 / 2} \frac{d r}{r}-\pi$

and

$$
J\left(r_{0}\right)=r_{0} \sqrt{\frac{D\left(r_{0}\right)}{B\left(r_{0}\right)}}
$$

For $D(r)=1$, equations (6) and (7) yield the results obtained by Weinberg [24].

The most general static and spherically symmetric solution to the Einstein massless scalar equations was independently obtained by Janis, Newman and Winicour as well as Wyman [25]. As both solutions were available in different coordinates, they were not known to be the same until Virbhadra [21] showed the equivalence between the two by a coordinate transformation. As Janis, Newman and Winicour obtained this solution about thirteen years before Wyman, we prefer to call it the Janis-NewmanWinicour solution. Thus, the Janis-Newman-Winicour solution (characterized by constant and real parameters, the ADM mass $M$ and the scalar charge $q$ ) is expressed by the line element

$$
\begin{aligned}
d s^{2} & =\left(1-\frac{b}{r}\right)^{\nu} d t^{2}-\left(1-\frac{b}{r}\right)^{-\nu} d r^{2} \\
& -\left(1-\frac{b}{r}\right)^{1-\nu} r^{2}\left(d \vartheta^{2}+\sin ^{2} \vartheta d \varphi^{2}\right)
\end{aligned}
$$

and the massless scalar field

$$
\Phi=\frac{q}{b \sqrt{4 \pi}} \ln \left(1-\frac{b}{r}\right)
$$

with

$$
\nu=\frac{2 M}{b} \quad \text { and } \quad b=2 \sqrt{M^{2}+q^{2}} .
$$


This solution is asymptotically Minkowskian and reduces to the Schwarzschild solution for $q=0$ (i.e., $\nu=1$ ). The Janis-Newman-Winicour solution has a globally naked strong curvature singularity at $r=b$ for all values of $q \neq 0$ and this solution is physically reasonable as it satisfies the weak energy condition [22]. Virbhadra et al. obtained the light deflection angle $\hat{\alpha}\left(r_{0}\right)$ for large value of $r_{0}$ (see equation (24) in [4]); we now re-express that using the equation (10), as follows:

$$
\begin{aligned}
\hat{\alpha}\left(r_{0}\right)=2 \nu\left(\frac{b}{r_{0}}\right) & +\left[\nu(1-2 \nu)+\pi\left(\nu^{2}-\frac{1}{16}\right)\right]\left(\frac{b}{r_{0}}\right)^{2} \\
& +\mathcal{O}\left(\frac{b}{r_{0}}\right)^{3} .
\end{aligned}
$$

Virbhadra and Ellis [5] as well as Claudel et al. [6] gave two different definitions of a photon sphere in a static spherically symmetric spacetime. Both definitions gave the same results for a general static and spherically symmetric metric. Thus, according to both definitions, the Janis-Newman-Winicour spacetime has only one photon sphere and that is situated at the radial distance [5, 6]

$$
r_{p s}=\frac{b(1+2 \nu)}{2}
$$

As $r=b$ is the curvature singularity, the photon sphere exists only for $\nu: 1 / 2<\nu \leq 1$.

Defining

$$
\rho=\frac{r}{b}, \quad \rho_{0}=\frac{r_{0}}{b}
$$

and using equations (6), (7) and (8), the deflection angle $\hat{\alpha}$ and the impact parameter $J$ for a light ray in the JanisNewman-Winicour spacetime are expressed in the form [4, 5]

$$
\hat{\alpha}\left(\rho_{0}\right)=2 \int_{\rho_{0}}^{\infty} \frac{d \rho}{\rho \sqrt{1-\frac{1}{\rho}} \sqrt{\left(\frac{\rho}{\rho_{0}}\right)^{2}\left(1-\frac{1}{\rho}\right)^{1-2 \nu}\left(1-\frac{1}{\rho_{0}}\right)^{2 \nu-1}-1}}-\pi
$$

and

$$
J\left(\rho_{0}\right)=2 M \frac{\rho_{0}}{\nu}\left(1-\frac{1}{\rho_{0}}\right)^{\frac{1-2 \nu}{2}} .
$$

Obviously, equation (12) can now be re-expressed as

$$
\rho_{p s}=\frac{(1+2 \nu)}{2} \text {. }
$$

Equation (15) with the expression for the impact parameter, $J=D_{d} \sin \theta$, give

$$
\sin \theta=\frac{2 M}{D_{d}} \frac{\rho_{0}}{\nu}\left(1-\frac{1}{\rho_{0}}\right)^{\frac{1-2 \nu}{2}} .
$$

The first derivative of the deflection angle $\hat{\alpha}$ with respect to $\theta$ is given by [4, 7]

$$
\frac{d \hat{\alpha}}{d \theta}=\hat{\alpha}^{\prime}\left(\rho_{0}\right) \frac{d \rho_{0}}{d \theta}
$$

where

$\frac{d \rho_{0}}{d \theta}=\frac{\nu \rho_{0}\left(1-\frac{1}{\rho_{0}}\right)^{\frac{1+2 \nu}{2}} \sqrt{1-\frac{4}{\nu^{2}}\left(\frac{M}{D_{d}}\right)^{2} \rho_{0}^{2}\left(1-\frac{1}{\rho_{0}}\right)^{1-2 \nu}}}{\frac{M}{D_{d}}\left(2 \rho_{0}-2 \nu-1\right)}$

and

$$
\hat{\alpha}^{\prime}\left(\rho_{0}\right)=\frac{2 \nu+1-2 \rho_{0}}{\rho_{0}^{2}\left(1-\frac{1}{\rho_{0}}\right)} \int_{\rho_{0}}^{\infty} \frac{(4 \nu \rho-2 \nu-1) d \rho}{(2 \nu+1-2 \rho)^{2} \rho \sqrt{1-\frac{1}{\rho}} \sqrt{\left(\frac{\rho}{\rho_{0}}\right)^{2}\left(1-\frac{1}{\rho}\right)^{1-2 \nu}\left(1-\frac{1}{\rho_{0}}\right)^{2 \nu-1}-1}} .
$$

The prime denotes the first derivative with respect to $\rho_{0}$.

Virbhadra and Ellis [7] classified naked singularities in two groups: Weakly naked singularities (WNS) are those which are contained within at least one photon sphere, whereas strongly naked singularities (SNS) are those which are not covered within any photon spheres. Therefore, according to this classification, the JanisNewman-Winicour naked singularities are strongly naked 
for $0 \leq \nu \leq 1 / 2$ and weakly naked for $1 / 2<\nu<1$.

For Schwarzschild black holes $(\nu=1)$ as well as WNS $(1 / 2<\nu<1)$, the deflection angle $\hat{\alpha}\left(\rho_{0}\right)$ monotonically increases with the decrease in the closest distance of approach $\rho_{0}$ and $\hat{\alpha}\left(\rho_{0}\right) \rightarrow \infty$ as $\rho_{0} \rightarrow \rho_{p s}$. As both have qualitatively similar $\hat{\alpha}$ vs. $\rho_{0}$ graph, their lensing features are also qualitatively similar [5, 7]. However, Virbhadra and Ellis [7] missed noticing a point: Though there are no photon spheres for $\nu=1 / 2$, the deflection angle behavior, according to the equation (20), is similar to the cases of the Schwarzschild black holes and WNS; therefore, their gravitational lensing features will be also qualitatively the same. In view of this, we now prefer to term $\nu=1 / 2$ and $0 \leq \nu<1 / 2$ singularities, respectively, marginally strongly naked singularities (MSNS) and strongly naked singularties.

The mass parameter $M=0$ in the Janis-NewmanWinicour solution describes the situation of a purely scalar field. We do not consider this case henceforth in this paper.

\section{TIME DELAY, MAGNIFICATION CENTROID, AND TOTAL MAGNIFICATION}

We consider light propagation in a static spherically symmetric spacetime described by the line element given by Eq. (5). The spherical symmetry of the spacetime allows us to consider, without loss of generality, null geodesics in the equatorial plane. We first obtain time required for light to travel from a source at coordinates $\left\{r, \vartheta=\pi / 2, \varphi=\varphi_{1}\right\}$ to the closest distance of approach at coordinates $\left\{r_{0}, \vartheta=\pi / 2, \varphi=\varphi_{2}\right\}$. Following the method used in [24], a straightforward calculation thus gives the time required for light to travel from $r$ to $r_{0}$ (or $r_{0}$ to $r$ ) that is expressed by

$$
t\left(r, r_{0}\right)=t\left(r_{0}, r\right)=\int_{r_{0}}^{r} \sqrt{\frac{A(r) / B(r)}{1-\left(\frac{r_{0}}{r}\right)^{2} \frac{B(r)}{B\left(r_{0}\right)} \frac{D\left(r_{0}\right)}{D(r)}}} d r .
$$

$D(r)=1$ in the above equation readily gives the result in [24]. Let $\mathcal{R}_{s}$ and $\mathcal{R}_{o}$ denote, respectively, the radial coordinates of the source and the observer measured from the center of mass of the deflector (lens). We now express these distances in terms of the constant parameter $b$ (in the Janis-Newman-Winicour metric) by introducing

$$
\mathcal{X}_{s}=\frac{\mathcal{R}_{s}}{b} \quad \text { and } \quad \mathcal{X}_{o}=\frac{\mathcal{R}_{o}}{b} .
$$

The time delay $\tau\left(\rho_{0}\right)$ of light traveling from the source to the observer with the closest of approach $\rho_{0}$ is defined as the difference between the light travel time for the actual ray in the gravitational field of the lens (deflector) and the travel time for the straight path between the source and the observer in the absence of the lens (i.e., if there were no gravitational fields.) As mentioned in Section II. we do not consider the case of purely scalar field in this paper; therefore, we assume that $\nu \neq 0$ (i.e., $M \neq 0$ ). Using equations (8), (10) and (21), and the geometry of the lens diagram (see Fig. 1 in [5]), we obtain the following expression for the time delay in the JanisNewman-Winicour spacetime:

$$
\tau\left(\rho_{0}\right)=\frac{2 M}{\nu}\left[\int_{\rho_{0}}^{\mathcal{X}_{s}} \frac{d \rho}{f(\rho)}+\int_{\rho_{0}}^{\mathcal{X}_{o}} \frac{d \rho}{f(\rho)}\right]-D_{s} \sec \beta
$$

with

$$
\begin{aligned}
& \mathcal{X}_{s}=\frac{\nu}{2} \frac{D_{s}}{M} \sqrt{\left(\frac{D_{d s}}{D_{s}}\right)^{2}+\tan ^{2} \beta}, \\
& \mathcal{X}_{o}=\frac{\nu}{2} \frac{D_{d}}{M}
\end{aligned}
$$

and

$$
f(\rho)=\sqrt{\left(1-\frac{1}{\rho}\right)^{2 \nu}-\left(\frac{\rho_{0}}{\rho}\right)^{2}\left(1-\frac{1}{\rho}\right)^{4 \nu-1}\left(1-\frac{1}{\rho_{0}}\right)^{1-2 \nu}} .
$$

The first and second terms in Eq. (23) give, respectively, the travel time of the light from the source to the point of closest approach and from that point to the observer. The last term gives the light travel time from the source to the observer in the absence of any gravitational field.

We use the equation (23) for computations in the next section. However, to see the behavior of the time delay function for a light ray traveling in the weak gravitation field far away from the lens, we carry out some analytical calculations following the method used in [15]. We obtain the time delay for images with large impact parameters. For given angular positions of the source and image, the time delay is given by

$\tau(\theta, \beta)=\frac{1}{2} \frac{D_{d} D_{s}}{D_{d s}}\left[\left(2-\frac{1}{\nu}\right) \theta_{E}^{2}+\beta^{2}-\theta^{2}-\ln \left(\frac{\theta^{2} D_{d}}{4 D_{d s}}\right)\right]$,

where

$$
\theta_{E}=\sqrt{4 M \frac{D_{d s}}{D_{d} D_{s}}}
$$

is an approximate expression for angular radius of the Einstein ring of Schwarzschild lensing.

For the direct image, $\beta^{2}-\theta^{2}$ decreases with an increase in $|\beta|$. Therefore, for $\nu<1 / 2$ (SNS), the time delay of the direct image is negative for large $\beta$. This fact reflects in our computations in the next section.

We denote the time delay in the outermost image on the same side as the source (also called the direct image) by $\tau_{o s}$. The differential time delay $\Delta \tau$ of an image with time delay $\tau$ is defined by

$$
\Delta \tau=\tau-\tau_{o s}
$$

(When there is only one image on the same side as the source, we use the symbol $\tau_{s}$ instead of $\tau_{o s}$.) The differential time delay is thus measured in reference to the direct image. The magnification-weighted centroid position 
(also called magnification centroid) of images is defined by

$$
\hat{\Theta}=\frac{\sum \theta_{i}\left|\mu_{i}\right|}{\sum\left|\mu_{i}\right|} .
$$

Angles measured in clockwise and anti-clockwise directions from the optical axis have positive and negative signs, respectively. Further, the magnification centroid shift of images is defined by

$$
\Delta \hat{\Theta}=\beta-\hat{\Theta} .
$$

The total absolute magnification (also, simply called total magnification) $\mu_{t o t}$ is defined by

$$
\mu_{t o t}=\sum\left|\mu_{i}\right| .
$$

The magnification centroid and the total magnification are very important physical quantities in studying microlensing when the images are not resolved.

\section{COMPUTATIONS}

Virbhadra and Ellis [7] modeled massive dark objects (MDOs) at the centers of four different galaxies (including our galaxy) as Schwarzschild black holes (SBH) and Janis-Newman-Winicour naked singularities, and studied point source gravitational lensing by them. They obtained the angular positions of critical curves and caustics, and studied the variation of magnification against the angular position of images near the critical curves. However, they did not study the time delay, magnification centroid, and the total magnification; we accomplish these tasks in this paper.

We now consider the MDO at the center of our galaxy with the recent values for the mass $M=3.61 \times 10^{6} M_{\odot}$ and the lens-observer distance $D_{d}=7.62 \mathrm{kpc}[26]$. As in 7], we take the lens (MDO) to be situated half way between the source and the observer, i.e., $D_{d s} / D_{s}=1 / 2$. We model the Galactic MDO as a Schwarzschild black hole as well as Janis-Newman-Winicour WNS, MSNS, and SNS lenses. As we are considering updated values for $M$ and $D_{d}$ of the Galactic MDO, we first re-compute critical curves and caustics, and their corresponding deflection angles of the light ray for several values of $\nu$. For continuity and clarity in the analysis of the results, we will also mention some results from [4, 7] in the present and next sections of this paper.

The existence of a photon sphere covering a gravitational lens is a sufficient (but not necessary) condition for the occurrence of relativistic images. The SBH as well as WNS both are contained inside a photon sphere and both give rise to relativistic images. The MSNS lensing also produces relativistic images, even though it is not covered by a photon sphere. It is known that relativistic images are transient and extremely demagnified, and therefore their observations do not seem to be feasible in the near future [5]. Hence, we do not do computations for relativistic images in this paper.

As shown in Table I, there is only one Einstein ring and no RCC for the case of SBH $(\nu=1)$, WNS $(\nu=$ $0.9,0.8,0.7,0.6)$, and MSNS $(\nu=0.5)$. The angular positions of the Einstein rings decrease very slowly with a decrease in the value of $\nu$ (equivalently, an increase in the value of $\left.(q / M)^{2}\right)$. In the case of SNS lensing $(0 \leq \nu<1 / 2)$, there are two situations: There is always one RCC; however, there can be two (for example, for $\nu=0.4,0.3$, etc.) or none Einstein rings (for example, for $\nu=0.001$ and any lower but nonnegative values of $\nu$ ). For the cases of double Einstein rings, the angular radii of outer and inner rings, respectively, decrease and increase with a decrease in the value of $\nu$. For a detailed analysis of these RCCs and TCCs, see [7].

We now compute image positions, the corresponding deflection angles of light, magnifications, time delays, and differential time delays for several values of the angular source position for different values of $\nu=1(\mathrm{SBH}), \nu=$ 0.7 (WNS), $\nu=0.5$ (MSNS), and $\nu=0.04,0.02,0.001$ (SNS). Though we give only a few data in the tables, we have computed and used many more data points for the figures.

The gravitational lensing effects due to the SBH, WNS, and MSNS are qualitatively similar, though they differ quantitatively by small values (see Tables II through IV and Figure 1). For each case, there are two images, one on each side of the optical axis. As the source moves away from the optical axis, the image on the same side as the source (i.e., the direct image) moves away from the axis, whereas the image on the opposite side of the source moves toward the axis. The absolute magnification of both images decreases. For a given value of $\nu$, with an increase in the angular position of the source, the time delays of the images on the same side as the source decrease, whereas the time delays and differential time delays of the image on the opposite side from the source increase. The rate of decrease in time delay of the image on the same side as the source is much slower than the rate of increase in time delay of the image on the opposite side from the source. For any given value of the angular source position, the time delays of both images and the differential time delay of the image on the opposite side from the source decrease with a decrease in the value of $\nu$ (equivalently, increase in $(q / M)^{2}$ ).

Gravitational lensing by a SNS is qualitatively very different from lensing by SBH, WNS, or MSNS (see Tables $\mathrm{V}$ through IX and Figures 2 through 4). For $\nu=0.04$ and 0.02 , when the lens components (the source, lens and observer) are perfectly (or nearly) aligned, there are two concentric Einstein rings (the inner ring much fainter than the outer one). As the alignment is broken, the two Einstein rings break into four images, two images on each side of the optical axis. The time delay of direct image decreases with an increase in $\beta$. For $\nu=0.04$, the time delay of the direct image is positive for small $\beta$ and negative for large $\beta$; however, the time delays for other images 
TABLE I: Critical curves and caustics due to gravitational lensing by the Galactic MDO modeled as the Schwarzschild black hole; weakly, marginally strongly, and strongly naked singularities. $\theta_{E}, \theta_{r}$, and $\beta_{r}$ denote respectively the angular positions of the tangential critical curves (Einstein rings), radial critical curves, and radial caustics, whereas $\hat{\alpha}$ stands for the corresponding light deflection angles. (a) The lens has mass $M=3.61 \times 10^{6} M_{\odot}$ and the distance $D_{d}=7.62 \mathrm{kpc}$ so that $M / D_{d} \approx 2.26 \times 10^{-11}$ ). The ratio of the source-lens distance to the source-observer distance, i.e., $D_{d s} / D_{s}=1 / 2$. All angular positions are given in arcseconds.

\begin{tabular}{|c|c|c|c|c|c|c|c|}
\hline \multirow[t]{2}{*}{$\nu$} & \multicolumn{2}{|c|}{ Inner Einstein ring } & \multicolumn{3}{|c|}{ Radial critical curve and caustic } & \multicolumn{2}{|c|}{ Outer Einstein ring } \\
\hline & $\theta_{E}$ & $\hat{\alpha}$ & $\theta_{r}$ & $\hat{\alpha}$ & $\beta_{r}$ & $\theta_{E}$ & $\hat{\alpha}$ \\
\hline 1.0 & $\times$ & $\bar{x}$ & $\times$ & $\bar{x}$ & $\times$ & 1.388176 & 2.776352 \\
\hline 0.9 & $x$ & $\times$ & $\times$ & $x$ & $x$ & 1.388176 & 2.776352 \\
\hline 0.8 & $\times$ & $\times$ & $\times$ & $\times$ & $\times$ & 1.388176 & 2.776352 \\
\hline 0.7 & $\times$ & $x$ & $\times$ & $x$ & $x$ & 1.388176 & 2.776351 \\
\hline 0.6 & $\times$ & $\times$ & $\times$ & $\times$ & $\times$ & 1.388175 & 2.776351 \\
\hline 0.5 & $x$ & $x$ & $\times$ & $x$ & $\times$ & 1.388175 & 2.776350 \\
\hline 0.4 & 0.000012 & 0.000024 & -0.000019 & 286883.8 & 252026.7 & 1.388174 & 2.776348 \\
\hline 0.3 & 0.000015 & 0.000030 & -0.000026 & 121357.9 & 66413.69 & 1.388172 & 2.776343 \\
\hline 0.2 & 0.000027 & 0.000054 & -0.000051 & 46469.19 & 23533.19 & 1.388165 & 2.776330 \\
\hline 0.1 & 0.000095 & 0.000191 & -0.000188 & 10759.36 & 5383.343 & 1.388131 & 2.776262 \\
\hline 0.05 & 0.000371 & 0.000741 & -0.000739 & 2641.816 & 1320.961 & 1.387993 & 2.775986 \\
\hline 0.04 & 0.000577 & 0.001154 & -0.001151 & 1687.159 & 843.5924 & 1.387890 & 2.775780 \\
\hline 0.03 & 0.001023 & 0.002046 & -0.002043 & 947.4574 & 473.7291 & 1.387667 & 2.775334 \\
\hline 0.02 & 0.002297 & 0.004593 & -0.004591 & 420.5955 & 210.2934 & 1.387029 & 2.774057 \\
\hline 0.01 & 0.009176 & 0.018352 & -0.018345 & 105.0746 & 52.51893 & 1.383568 & 2.767135 \\
\hline 0.005 & 0.036717 & 0.073434 & -0.073177 & 26.26379 & 13.05872 & 1.369456 & 2.738911 \\
\hline 0.004 & 0.057426 & 0.114853 & -0.113887 & 16.80784 & 8.290033 & 1.358574 & 2.717148 \\
\hline 0.003 & 0.102472 & 0.204944 & -0.199693 & 9.450634 & 4.525624 & 1.334103 & 2.668206 \\
\hline 0.002 & 0.236134 & 0.472268 & -0.420122 & 4.166782 & 1.663269 & 1.254966 & 2.509932 \\
\hline 0.001 & $x$ & $\times$ & 1.115015 & 0.613237 & 0.808397 & $\times$ & $x$ \\
\hline
\end{tabular}

TABLE II: Image positions due to lensing by the Galactic MDO modeled as the Schwarzschild black hole $(\nu=1)$, and their respective bending angles, magnifications, time delays, and differential time delays. (a) $\theta$ and $\hat{\alpha}$ respectively stand for the angular positions of images and their corresponding deflection angles. $\mu, \tau$, and $\Delta \tau$ represent the magnification, time delay, and differential time delay of the images, respectively. (b) The subscripts $s$ and $o$ on the symbols respectively denote for the images on the same and opposite side of the source. (c) The same as (a) of Table I.

\begin{tabular}{|c|c|c|c|c|c|c|c|c|c|}
\hline \multirow[t]{2}{*}{$\bar{\beta}$} & \multicolumn{5}{|c|}{ Image on the opposite side from the source } & \multicolumn{4}{|c|}{ Image on the same side as the source } \\
\hline & $\theta_{0}$ & $\hat{\alpha}_{o}$ & $\mu_{o}$ & $\tau_{o}$ & $\Delta \tau_{o}$ & $\theta_{s}$ & $\hat{\alpha}_{s}$ & $\mu_{s}$ & $\tau_{s}$ \\
\hline 0 & -1.388176 & 2.776352 & $\bar{x}$ & 14.92209 & 0 & 1.388176 & 2.776352 & $\bar{x}$ & 14.92209 \\
\hline $10^{-6}$ & -1.388176 & 2.776353 & -694084.2 & 14.92209 & 0.000002 & 1.388177 & 2.776351 & 694085.2 & 14.92209 \\
\hline $10^{-5}$ & -1.388171 & 2.776362 & -69407.97 & 14.92210 & 0.000017 & 1.388181 & 2.776342 & 69408.97 & 14.92208 \\
\hline $10^{-4}$ & -1.388126 & 2.776452 & -6940.347 & 14.92218 & 0.000171 & 1.388226 & 2.776252 & 6941.347 & 14.92201 \\
\hline $10^{-3}$ & -1.387676 & 2.777353 & -693.5848 & 14.92294 & 0.001706 & 1.388676 & 2.775353 & 694.5848 & 14.92124 \\
\hline $10^{-2}$ & -1.383185 & 2.786370 & -68.90982 & 14.93064 & 0.017060 & 1.393185 & 2.766370 & 69.90982 & 14.91358 \\
\hline $10^{-1}$ & -1.339077 & 2.878153 & -6.454348 & 15.00895 & 0.170636 & 1.439076 & 2.678152 & 7.454345 & 14.83831 \\
\hline 1 & -0.975480 & 3.950960 & -0.322455 & 15.94681 & 1.742193 & 1.975475 & 1.950951 & 1.322453 & 14.20461 \\
\hline 2 & -0.710863 & 5.421726 & -0.073840 & 17.38033 & 3.687537 & 2.710855 & 1.421709 & 1.073838 & 13.69280 \\
\hline 3 & -0.543786 & 7.087573 & -0.024114 & 19.29818 & 5.987040 & 3.543776 & 1.087553 & 1.024113 & 13.31114 \\
\hline 4 & -0.434559 & 8.869117 & -0.009696 & 21.74718 & 8.734391 & 4.434547 & 0.869094 & 1.009695 & 13.01279 \\
\hline 5 & -0.359561 & 10.71912 & -0.004521 & 24.75495 & 11.98479 & 5.359549 & 0.719098 & 1.004521 & 12.77016 \\
\hline 6 & -0.305617 & 12.61123 & -0.002355 & 28.33806 & 15.77141 & 6.305604 & 0.611208 & 1.002354 & 12.56665 \\
\hline 7 & -0.265251 & 14.53050 & -0.001335 & 32.50696 & 20.11513 & 7.265238 & 0.530476 & 1.001335 & 12.39183 \\
\hline 8 & -0.234044 & 16.46809 & -0.000809 & 37.26852 & 25.02969 & 8.234031 & 0.468062 & 1.000808 & 12.23882 \\
\hline 9 & -0.209261 & 18.41852 & -0.000517 & 42.62746 & 30.52455 & 9.209248 & 0.418496 & 1.000517 & 12.10291 \\
\hline 10 & -0.189138 & 20.37828 & -0.000345 & 48.58714 & 36.60642 & 10.18912 & 0.378250 & 1.000345 & 11.98072 \\
\hline
\end{tabular}

are positive for all values of $\beta$. On the other hand, for $\nu=0.02$, the time delay of the direct image is always negative; however, the other three images have negative time delays for small $\beta$ and positive for large $\beta$, passing through the zero time delay point.

For a given value of $\beta$, the time delays of images are in the following decreasing order: the inner image on the opposite side from the source, inner image on the same side as the source, outer image on the opposite side from the source, and the direct image. However, for any given value of $\beta$, the absolute magnifications of images are not in the exact reverse sequence; they are rather in the following decreasing order: The direct image, outer image on the opposite side from the source, inner image on the opposite side from the source, and inner image on the same side as the source. The differential time delays are always positive. It is worth emphasizing that the negative and positive time delays are respectively not necessarily due to negative and positive bending angles. A light ray with a positive deflection angle may give rise to positive or negative time delays, and the same is true for a light ray with a negative deflection angle. If $\beta$ increases, the angular separation between images on the same side as the source increases (the inner and outer images move respectively toward and away from the optical axis). However, the angular separation between images on the op- 
TABLE III: Image positions due to lensing by the Galactic MDO modeled as the weakly naked singularity $(\nu=0.7)$, and their respective bending angles, magnifications, time delays and differential time delays. (a) The same as (a) and (b) of Table II. (b) The same as (a) of Table I.

\begin{tabular}{|c|c|c|c|c|c|c|c|c|c|}
\hline \multirow[t]{2}{*}{$\bar{\beta}$} & \multicolumn{5}{|c|}{ Image on the opposite side from the source } & \multicolumn{4}{|c|}{ Image on the same side as the source } \\
\hline & $\theta_{o}$ & $\hat{\alpha}_{o}$ & $\mu_{o}$ & $\tau_{o}$ & $\Delta \tau_{o}$ & $\theta_{s}$ & $\hat{\alpha}_{s}$ & $\mu_{s}$ & $\tau_{s}$ \\
\hline 0 & -1.388176 & 2.776351 & $\times$ & 14.66836 & 0 & 1.388176 & 2.776351 & $x$ & 14.66836 \\
\hline $10^{-6}$ & -1.388175 & 2.776352 & -694084.2 & 14.66836 & 0.000002 & 1.388176 & 2.776350 & 694085.2 & 14.66835 \\
\hline $10^{-5}$ & -1.388171 & 2.776361 & -69407.97 & 14.66836 & 0.000017 & 1.388181 & 2.776341 & 69408.97 & 14.66835 \\
\hline $10^{-4}$ & -1.388126 & 2.776451 & -6940.347 & 14.66844 & 0.000171 & 1.388226 & 2.776251 & 6941.347 & 14.66827 \\
\hline $10^{-3}$ & -1.387676 & 2.777352 & -693.5848 & 14.66921 & 0.001706 & 1.388676 & 2.775352 & 694.5848 & 14.66750 \\
\hline $10^{-2}$ & -1.383185 & 2.786370 & -68.90982 & 14.67690 & 0.017060 & 1.393185 & 2.766369 & 69.90982 & 14.65984 \\
\hline $10^{-1}$ & -1.339076 & 2.878152 & -6.454347 & 14.75521 & 0.170636 & 1.439076 & 2.678151 & 7.454345 & 14.58457 \\
\hline 1 & -0.975479 & 3.950959 & -0.322455 & 15.69307 & 1.742192 & 1.975475 & 1.950950 & 1.322453 & 13.95088 \\
\hline 2 & -0.710862 & 5.421724 & -0.073840 & 17.12660 & 3.687536 & 2.710855 & 1.421709 & 1.073838 & 13.43906 \\
\hline 3 & -0.543786 & 7.087571 & -0.024113 & 19.04444 & 5.987039 & 3.543776 & 1.087552 & 1.024113 & 13.05740 \\
\hline 4 & -0.434558 & 8.869115 & -0.009696 & 21.49345 & 8.734389 & 4.434547 & 0.869094 & 1.009695 & 12.75906 \\
\hline 5 & -0.359560 & 10.71912 & -0.004521 & 24.50121 & 11.98478 & 5.359549 & 0.719097 & 1.004521 & 12.51643 \\
\hline 6 & -0.305616 & 12.61123 & -0.002355 & 28.08432 & 15.77141 & 6.305604 & 0.611208 & 1.002354 & 12.31292 \\
\hline 7 & -0.265250 & 14.53050 & -0.001335 & 32.25322 & 20.11513 & 7.265238 & 0.530476 & 1.001335 & 12.13810 \\
\hline 8 & -0.234043 & 16.46809 & -0.000809 & 37.01478 & 25.02969 & 8.234031 & 0.468062 & 1.000808 & 11.98509 \\
\hline 9 & -0.209260 & 18.41852 & -0.000517 & 42.37372 & 30.52454 & 9.209248 & 0.418496 & 1.000517 & 11.84918 \\
\hline 10 & -0.189137 & 20.37827 & -0.000345 & 48.33340 & 36.60642 & 10.18912 & 0.378250 & 1.000345 & 11.72698 \\
\hline
\end{tabular}

TABLE IV: Image positions due to lensing by the Galactic MDO modeled as the marginally strongly naked singularity $(\nu=0.5)$ and their respective bending angles, magnifications, time delays and differential time delays. (a) The same as (a) and (b) of Table II. (b) The same as (a) of Table I.

\begin{tabular}{|c|c|c|c|c|c|c|c|c|c|}
\hline \multirow[t]{2}{*}{$\beta$} & \multicolumn{5}{|c|}{ Image on the opposite side from the source } & \multicolumn{4}{|c|}{ Image on the same side as the source } \\
\hline & $\theta_{0}$ & $\hat{\alpha}_{o}$ & $\mu_{o}$ & $\tau_{o}$ & $\Delta \tau_{o}$ & $\theta_{s}$ & $\hat{\alpha}_{s}$ & $\mu_{s}$ & $\tau_{s}$ \\
\hline 0 & -1.388175 & 2.776350 & $\bar{x}$ & 14.33004 & 0 & 1.388175 & 2.776350 & $x$ & 14.33004 \\
\hline $10^{-6}$ & -1.388174 & 2.776351 & -694084.2 & 14.33004 & 0.000002 & 1.388175 & 2.776349 & 694085.2 & 14.33004 \\
\hline $10^{-5}$ & -1.388170 & 2.776360 & -69407.97 & 14.33005 & 0.000017 & 1.388180 & 2.776340 & 69408.97 & 14.33003 \\
\hline $10^{-4}$ & -1.388125 & 2.776450 & -6940.347 & 14.33013 & 0.000171 & 1.388225 & 2.776250 & 6941.347 & 14.32995 \\
\hline $10^{-3}$ & -1.387675 & 2.777350 & -693.5848 & 14.33089 & 0.001706 & 1.388675 & 2.775350 & 694.5848 & 14.32919 \\
\hline $10^{-2}$ & -1.383184 & 2.786368 & -68.90982 & 14.33859 & 0.017060 & 1.393184 & 2.766368 & 69.90982 & 14.32153 \\
\hline $10^{-1}$ & -1.339075 & 2.878150 & -6.454347 & 14.41689 & 0.170636 & 1.439075 & 2.678150 & 7.454345 & 14.24626 \\
\hline 1 & -0.975478 & 3.950956 & -0.322455 & 15.35475 & 1.742191 & 1.975474 & 1.950949 & 1.322453 & 13.61256 \\
\hline 2 & -0.710861 & 5.421721 & -0.073840 & 16.78828 & 3.687533 & 2.710854 & 1.421708 & 1.073838 & 13.10075 \\
\hline 3 & -0.543784 & 7.087568 & -0.024113 & 18.70612 & 5.987035 & 3.543776 & 1.087552 & 1.024113 & 12.71909 \\
\hline 4 & -0.434556 & 8.869112 & -0.009696 & 21.15513 & 8.734385 & 4.434547 & 0.869094 & 1.009695 & 12.42074 \\
\hline 5 & -0.359558 & 10.71912 & -0.004521 & 24.16289 & 11.98478 & 5.359549 & 0.719097 & 1.004521 & 12.17811 \\
\hline 6 & -0.305614 & 12.61123 & -0.002354 & 27.74600 & 15.77140 & 6.305604 & 0.611208 & 1.002354 & 11.97460 \\
\hline 7 & -0.265248 & 14.53050 & -0.001335 & 31.91490 & 20.11512 & 7.265238 & 0.530476 & 1.001335 & 11.79978 \\
\hline 8 & -0.234041 & 16.46808 & -0.000809 & 36.67646 & 25.02968 & 8.234031 & 0.468062 & 1.000808 & 11.64678 \\
\hline 9 & -0.209259 & 18.41852 & -0.000517 & 42.03539 & 30.52453 & 9.209248 & 0.418496 & 1.000517 & 11.51086 \\
\hline 10 & -0.189135 & 20.37827 & -0.000345 & 47.99508 & 36.60640 & 10.18912 & 0.378250 & 1.000345 & 11.38867 \\
\hline
\end{tabular}

TABLE V: Image positions on the same side as the source due to lensing by the Galactic MDO modeled as a strongly naked singularity $(\nu=0.04)$, and their respective bending angles, magnifications, time delays and differential time delays. (a) The same as (a) of Table II. (b) The subscripts is and os on the symbols respectively denote for the inner and outer images on the same side as the source. (c) The same as (a) of Table I.

\begin{tabular}{|c|c|c|c|c|c|c|c|c|c|}
\hline \multirow[t]{2}{*}{$\beta$} & \multicolumn{5}{|c|}{ Inner image on the same side as the source } & \multicolumn{4}{|c|}{ Outer image on the same side as the source } \\
\hline & $\theta_{i s}$ & $\hat{\alpha}_{i s}$ & $\mu_{i s}$ & $\tau_{i s}$ & $\Delta \tau_{i s}$ & $\theta_{o s}$ & $\hat{\alpha}_{o s}$ & $\mu_{o s}$ & $\tau_{o s}$ \\
\hline $10^{-6}$ & 0.000577 & 0.001152 & -0.000099 & 8.164602 & 7.452654 & 1.387890 & 2.775779 & 694085.2 & 0.711948 \\
\hline $10^{-5}$ & 0.000577 & 0.001134 & $-9.9 \times 10^{-6}$ & 8.164602 & 7.452662 & 1.387895 & 2.775770 & 69408.97 & 0.711940 \\
\hline $10^{-4}$ & 0.000577 & 0.000954 & $-9.9 \times 10^{-7}$ & 8.164602 & 7.452738 & 1.387940 & 2.775680 & 6941.347 & 0.711863 \\
\hline $10^{-3}$ & 0.000577 & -0.000846 & $-9.9 \times 10^{-8}$ & 8.164602 & 7.453505 & 1.388390 & 2.774780 & 694.5849 & 0.711097 \\
\hline $10^{-2}$ & 0.000577 & -0.018846 & $-9.9 \times 10^{-9}$ & 8.164629 & 7.461187 & 1.392900 & 2.765800 & 69.90987 & 0.703442 \\
\hline $10^{-1}$ & 0.000577 & -0.198846 & $-9.9 \times 10^{-10}$ & 8.167637 & 7.539395 & 1.438800 & 2.677600 & 7.454397 & 0.628242 \\
\hline 1 & 0.000577 & -1.998846 & $-9.9 \times 10^{-11}$ & 8.471271 & 8.476159 & 1.975286 & 1.950572 & 1.322496 & -0.004887 \\
\hline 2 & 0.000577 & -3.998847 & $-4.9 \times 10^{-11}$ & 9.391988 & 9.908244 & 2.710736 & 1.421472 & 1.073866 & -0.516256 \\
\hline 3 & 0.000576 & -5.998847 & $-3.3 \times 10^{-11}$ & 10.92675 & 11.82434 & 3.543700 & 1.087400 & 1.024129 & -0.897592 \\
\hline 4 & 0.000576 & -7.998848 & $-2.5 \times 10^{-11}$ & 13.07556 & 14.27126 & 4.434496 & 0.868992 & 1.009705 & -1.195693 \\
\hline 5 & 0.000576 & -9.998848 & $-2.0 \times 10^{-11}$ & 15.83842 & 17.27655 & 5.359513 & 0.719026 & 1.004527 & -1.438130 \\
\hline 6 & 0.000576 & -11.99885 & $-1.6 \times 10^{-11}$ & 19.21533 & 20.85681 & 6.305578 & 0.611155 & 1.002358 & -1.641480 \\
\hline 7 & 0.000576 & -13.99885 & $-1.4 \times 10^{-11}$ & 23.20628 & 25.02245 & 7.265218 & 0.530436 & 1.001337 & -1.816167 \\
\hline 8 & 0.000576 & -15.99885 & $-1.2 \times 10^{-11}$ & 27.81128 & 29.78034 & 8.234015 & 0.468030 & 1.000810 & -1.969056 \\
\hline 9 & 0.000575 & -17.99885 & $-1.1 \times 10^{-11}$ & 33.03033 & 35.13520 & 9.209235 & 0.418471 & 1.000518 & -2.104868 \\
\hline 10 & 0.000575 & -19.99885 & $-9.7 \times 10^{-12}$ & 38.86343 & 41.09040 & 10.18911 & 0.378229 & 1.000346 & -2.226966 \\
\hline
\end{tabular}


TABLE VI: Image positions on the opposite side of the source due to lensing by the Galactic MDO modeled as a strongly naked singularity $(\nu=0.04)$, and their respective bending angles, magnifications, time delays and differential time delays. (a) The same as (a) of Table II. (b) The subscripts $i o$ and oo on the symbols respectively denote for the inner and outer images on the opposite side from the source. (c) The same as (a) of Table I.

\begin{tabular}{|c|c|c|c|c|c|c|c|c|c|c|}
\hline \multirow[t]{2}{*}{$\beta$} & \multicolumn{5}{|c|}{ Outer image on the opposite side from the source } & \multicolumn{5}{|c|}{ Inner image on the opposite side from the source } \\
\hline & $\theta_{\text {oo }}$ & $\hat{\alpha}_{o o}$ & $\mu_{o o}$ & $\tau_{o o}$ & $\Delta \tau_{o o}$ & $\theta_{i o}$ & $\hat{\alpha}_{i o}$ & $\mu_{i o}$ & $\tau_{i o}$ & $\Delta \tau_{i o}$ \\
\hline $10^{-6}$ & -1.387889 & 2.775781 & -694084.2 & 0.711950 & 0.000002 & -0.000577 & 0.001156 & 0.000099 & 8.164602 & 7.452654 \\
\hline $10^{-5}$ & -1.387885 & 2.775790 & -69407.97 & 0.711957 & 0.000017 & -0.000577 & 0.001174 & $9.9 \times 10^{-6}$ & 8.164602 & 7.452662 \\
\hline $10^{-4}$ & -1.387840 & 2.775880 & -6940.347 & 0.712034 & 0.000170 & -0.000577 & 0.001354 & $9.9 \times 10^{-7}$ & 8.164602 & 7.452738 \\
\hline $10^{-3}$ & -1.387390 & 2.776780 & -693.5848 & 0.712801 & 0.001704 & -0.000577 & 0.003154 & $9.9 \times 10^{-8}$ & 8.164603 & 7.453506 \\
\hline $10^{-2}$ & -1.382898 & 2.785796 & -68.90977 & 0.720486 & 0.017045 & -0.000577 & 0.021154 & $9.9 \times 10^{-9}$ & 8.164636 & 7.461194 \\
\hline $10^{-1}$ & -1.338780 & 2.877560 & -6.454297 & 0.798725 & 0.170483 & -0.000577 & 0.201154 & $9.9 \times 10^{-10}$ & 8.167708 & 7.539465 \\
\hline 1 & -0.975097 & 3.950193 & -0.322412 & 1.735749 & 1.740636 & -0.000577 & 2.001154 & $9.9 \times 10^{-11}$ & 8.471980 & 8.476867 \\
\hline 2 & -0.710409 & 5.420818 & -0.073812 & 3.168027 & 3.684283 & -0.000577 & 4.001155 & $5.0 \times 10^{-11}$ & 9.393405 & 9.909661 \\
\hline 3 & -0.543290 & 7.086579 & -0.024097 & 5.084252 & 5.981844 & -0.000577 & 6.001155 & $3.3 \times 10^{-11}$ & 10.92888 & 11.82647 \\
\hline 4 & -0.434037 & 8.868073 & -0.009686 & 7.531250 & 8.726943 & -0.000578 & 8.001155 & $2.5 \times 10^{-11}$ & 13.07840 & 14.27409 \\
\hline 5 & -0.359024 & 10.71805 & -0.004515 & 10.53661 & 11.97474 & -0.000578 & 10.00116 & $2.0 \times 10^{-11}$ & 15.84197 & 17.28010 \\
\hline 6 & -0.305070 & 12.61014 & -0.002351 & 14.11691 & 15.75839 & -0.000578 & 12.00116 & $1.7 \times 10^{-11}$ & 19.21958 & 20.86106 \\
\hline 7 & -0.264698 & 14.52939 & -0.001332 & 18.28259 & 20.09876 & -0.000578 & 14.00116 & $1.4 \times 10^{-11}$ & 23.21124 & 25.02741 \\
\hline 8 & -0.233486 & 16.46697 & -0.000807 & 23.04051 & 25.00957 & -0.000578 & 16.00116 & $1.2 \times 10^{-11}$ & 27.81695 & 29.78601 \\
\hline 9 & -0.208700 & 18.41740 & -0.000515 & 28.39540 & 30.50027 & -0.000578 & 18.00116 & $1.1 \times 10^{-11}$ & 33.03671 & 35.14158 \\
\hline 10 & -0.188575 & 20.37715 & -0.000344 & 34.35062 & 36.57758 & -0.000579 & 20.00116 & $1.0 \times 10^{-11}$ & 38.87051 & 41.09748 \\
\hline
\end{tabular}

TABLE VII: Image positions on the same side as the source due to lensing by the Galactic MDO modeled as a strongly naked singularity $(\nu=0.02)$, and their respective bending angles, magnifications, time delays and differential time delays. (a) The same as (a) of Table II. (b) The same as (b) of Table V. (c) The same as (a) of Table I.

\begin{tabular}{|c|c|c|c|c|c|c|c|c|c|}
\hline \multirow[t]{2}{*}{$\beta$} & \multicolumn{5}{|c|}{ Inner image on the same side as the source } & \multicolumn{4}{|c|}{ Outer image on the same side as the source } \\
\hline & $\theta_{\text {is }}$ & $\hat{\alpha}_{i s}$ & $\mu_{i s}$ & $\tau_{i s}$ & $\Delta \tau_{i s}$ & $\theta_{o s}$ & $\hat{\alpha}_{o s}$ & $\mu_{o s}$ & $\tau_{o s}$ \\
\hline 0 & 0.002297 & 0.004593 & $x$ & -8.271824 & 5.808667 & 1.387029 & 2.774057 & $\times$ & -14.08049 \\
\hline $10^{-6}$ & 0.002297 & 0.004591 & -0.006273 & -8.271824 & 5.808667 & 1.387029 & 2.774056 & 694085.9 & -14.08049 \\
\hline $10^{-5}$ & 0.002297 & 0.004573 & -0.000627 & -8.271824 & 5.808675 & 1.387034 & 2.774047 & 69409.04 & -14.08050 \\
\hline $10^{-4}$ & 0.002297 & 0.004393 & -0.000063 & -8.271824 & 5.808752 & 1.387079 & 2.773958 & 6941.354 & -14.08058 \\
\hline $10^{-3}$ & 0.002297 & 0.002593 & -0.000006 & -8.271825 & 5.809517 & 1.387529 & 2.773058 & 694.5857 & -14.08134 \\
\hline $10^{-2}$ & 0.002297 & -0.015407 & $-6.3 \times 10^{-7}$ & -8.271807 & 5.817185 & 1.392042 & 2.764084 & 69.91009 & -14.08899 \\
\hline $10^{-1}$ & 0.002296 & -0.195407 & $-6.3 \times 10^{-8}$ & -8.268894 & 5.895250 & 1.437970 & 2.675940 & 7.454558 & -14.16414 \\
\hline 1 & 0.002294 & -1.995412 & $-6.2 \times 10^{-9}$ & -7.966209 & 6.830681 & 1.974717 & 1.949434 & 1.322625 & -14.79689 \\
\hline 2 & 0.002291 & -3.995418 & $-3.1 \times 10^{-9}$ & -7.046546 & 8.261433 & 2.710378 & 1.420757 & 1.073949 & -15.30798 \\
\hline 3 & 0.002289 & -5.995423 & $-2.1 \times 10^{-9}$ & -5.512834 & 10.17630 & 3.543471 & 1.086943 & 1.024177 & -15.68914 \\
\hline 4 & 0.002286 & -7.995428 & $-1.5 \times 10^{-9}$ & -3.365073 & 12.62205 & 4.434343 & 0.868685 & 1.009733 & -15.98712 \\
\hline 5 & 0.002283 & -9.995434 & $-1.2 \times 10^{-9}$ & -0.603263 & 15.62622 & 5.359405 & 0.718809 & 1.004544 & -16.22948 \\
\hline 6 & 0.002280 & -11.99544 & $-1.0 \times 10^{-9}$ & 2.772596 & 19.20537 & 6.305498 & 0.610996 & 1.002370 & -16.43277 \\
\hline 7 & 0.002278 & -13.99544 & $-8.6 \times 10^{-10}$ & 6.762504 & 23.36992 & 7.265157 & 0.530315 & 1.001345 & -16.60742 \\
\hline 8 & 0.002275 & -15.99545 & $-7.5 \times 10^{-10}$ & 11.36646 & 28.12674 & 8.233968 & 0.467935 & 1.000816 & -16.76028 \\
\hline 9 & 0.002273 & -17.99545 & $-6.6 \times 10^{-10}$ & 16.58447 & 33.48053 & 9.209197 & 0.418394 & 1.000522 & -16.89606 \\
\hline 10 & 0.002270 & -19.99546 & $-5.9 \times 10^{-10}$ & 22.41652 & 39.43466 & 10.18908 & 0.378166 & 1.000349 & -17.01814 \\
\hline
\end{tabular}

TABLE VIII: Image positions on the opposite side of the source due to lensing by the Galactic MDO modeled as a strongly naked singularity $(\nu=0.02)$, and their respective bending angles, magnifications, time delays and differential time delays. (a) The same as (a) of Table II. (b) The same as (b) of Table VI. (c) The same as (a) of Table I.

\begin{tabular}{|c|c|c|c|c|c|c|c|c|c|c|}
\hline \multirow[t]{2}{*}{$\beta$} & \multicolumn{5}{|c|}{ Outer image on the opposite side from the source } & \multicolumn{5}{|c|}{ Inner image on the opposite side from the source } \\
\hline & $\theta_{o o}$ & $\hat{\alpha}_{o o}$ & $\mu_{o o}$ & $\tau_{o o}$ & $\Delta \tau_{o o}$ & $\theta_{i o}$ & $\hat{\alpha}_{i o}$ & $\mu_{i o}$ & $\tau_{i o}$ & $\Delta \tau_{i o}$ \\
\hline 0 & -1.387029 & 2.774057 & $\times$ & -14.08049 & 0 & -0.002297 & 0.004593 & $\times$ & -8.271824 & 5.808667 \\
\hline $10^{-6}$ & -1.387028 & 2.774058 & -694084.9 & -14.08049 & 0.000002 & -0.002297 & 0.004595 & 0.006273 & -8.271824 & 5.808667 \\
\hline $10^{-5}$ & -1.387024 & 2.774067 & -69408.04 & -14.08048 & 0.000017 & -0.002297 & 0.004613 & 0.000627 & -8.271824 & 5.808675 \\
\hline $10^{-4}$ & -1.386979 & 2.774157 & -6940.354 & -14.08041 & 0.000170 & -0.002297 & 0.004793 & 0.000063 & -8.271824 & 5.808752 \\
\hline $10^{-3}$ & -1.386528 & 2.775057 & -693.5853 & -14.07964 & 0.001703 & -0.002297 & 0.006593 & 0.000006 & -8.271822 & 5.809520 \\
\hline $10^{-2}$ & -1.382034 & 2.784067 & -68.90968 & -14.07196 & 0.017034 & -0.002297 & 0.024593 & $6.3 \times 10^{-7}$ & -8.271779 & 5.817213 \\
\hline $10^{-1}$ & -1.337888 & 2.875776 & -6.454149 & -13.99377 & 0.170377 & -0.002297 & 0.204594 & $6.3 \times 10^{-8}$ & -8.268612 & 5.895533 \\
\hline 1 & -0.973942 & 3.947885 & -0.322284 & -13.05731 & 1.739578 & -0.002299 & 2.004599 & $6.3 \times 10^{-9}$ & -7.963389 & 6.833501 \\
\hline 2 & -0.709041 & 5.418082 & -0.073730 & -11.62581 & 3.682166 & -0.002302 & 4.004604 & $3.2 \times 10^{-9}$ & -7.040905 & 8.267074 \\
\hline 3 & -0.541791 & 7.083582 & -0.024049 & -9.710471 & 5.978668 & -0.002305 & 6.004610 & $2.1 \times 10^{-9}$ & -5.504373 & 10.18477 \\
\hline 4 & -0.432460 & 8.864920 & -0.009657 & -7.264419 & 8.722705 & -0.002308 & 8.004615 & $1.6 \times 10^{-9}$ & -3.353791 & 12.63333 \\
\hline 5 & -0.357399 & 10.71480 & -0.004497 & -4.260046 & 11.96944 & -0.002310 & 10.00462 & $1.3 \times 10^{-9}$ & -0.589160 & 15.64032 \\
\hline 6 & -0.303414 & 12.60683 & -0.002339 & -0.680750 & 15.75203 & -0.002313 & 12.00463 & $1.1 \times 10^{-9}$ & 2.789520 & 19.22229 \\
\hline 7 & -0.263020 & 14.52604 & -0.001324 & 3.483905 & 20.09132 & -0.002316 & 14.00463 & $9.3 \times 10^{-10}$ & 6.782248 & 23.38967 \\
\hline 8 & -0.231793 & 16.46359 & -0.000801 & 8.240791 & 25.00107 & -0.002319 & 16.00464 & $8.2 \times 10^{-10}$ & 11.38903 & 28.14930 \\
\hline 9 & -0.206995 & 18.41399 & -0.000511 & 13.59463 & 30.49070 & -0.002322 & 18.00464 & $7.4 \times 10^{-10}$ & 16.60985 & 33.50591 \\
\hline 10 & -0.186860 & 20.37372 & -0.000341 & 19.54880 & 36.56694 & -0.002325 & 20.00465 & $6.7 \times 10^{-10}$ & 22.44473 & 39.46287 \\
\hline
\end{tabular}


TABLE IX: Image positions on the same side as the source due to lensing by the Galactic MDO modeled as the strongly naked singularity $(\nu=0.001)$, and their respective bending angles, magnifications, time delays and differential time delays. (a) The same as (a) of Table II. (b) The same as (b) of Table V. (c) The same as (a) of Table I.

\begin{tabular}{|c|c|c|c|c|c|c|c|c|c|}
\hline \multirow[t]{2}{*}{$\beta$} & \multicolumn{5}{|c|}{ Inner image on the same side as the source } & \multicolumn{4}{|c|}{ Outer image on the same side as the source } \\
\hline & $\theta_{i s}$ & $\hat{\alpha}_{i s}$ & $\mu_{i s}$ & $\tau_{i s}$ & $\Delta \tau_{i s}$ & $\theta_{o s}$ & $\hat{\alpha}_{o s}$ & $\mu_{o s}$ & $\tau_{o s}$ \\
\hline 0.75 & $\times$ & $\times$ & $\times$ & $\times$ & $\times$ & $\times$ & $\times$ & $\times$ & $\times$ \\
\hline 0.85 & 0.985460 & 0.270920 & -1.634301 & -577.4174 & 0.004886 & 1.273212 & 0.846424 & 3.146478 & -577.4223 \\
\hline 1 & 0.864695 & -0.270610 & -0.457456 & -577.4165 & 0.049078 & 1.498946 & 0.997892 & 1.854975 & -577.4656 \\
\hline 2 & 0.632971 & -2.734059 & -0.038933 & -576.9387 & 0.841932 & 2.488924 & 0.977849 & 1.150355 & -577.7807 \\
\hline 3 & 0.542216 & -4.915569 & -0.012362 & -575.7617 & 2.295525 & 3.412890 & 0.825779 & 1.056767 & -578.0572 \\
\hline 4 & 0.486179 & -7.027642 & -0.005625 & -573.9272 & 4.363376 & 4.349611 & 0.699223 & 1.026918 & -578.2905 \\
\hline 5 & 0.446365 & -9.107269 & -0.003071 & -571.4497 & 7.039872 & 5.300639 & 0.601278 & 1.014622 & -578.4895 \\
\hline 6 & 0.415912 & -11.16818 & -0.001874 & -568.3368 & 10.32523 & 6.262637 & 0.525273 & 1.008728 & -578.6620 \\
\hline 7 & 0.391513 & -13.21697 & -0.001234 & -564.5931 & 14.22061 & 7.232646 & 0.465292 & 1.005587 & -578.8137 \\
\hline 8 & 0.371325 & -15.25735 & -0.000859 & -560.2218 & 18.72713 & 8.208527 & 0.417054 & 1.003774 & -578.9489 \\
\hline 9 & 0.354221 & -17.29156 & -0.000623 & -555.2250 & 23.84570 & 9.188781 & 0.377562 & 1.002660 & -579.0707 \\
\hline 10 & 0.339464 & -19.32107 & -0.000467 & -549.6044 & 29.57700 & 10.17236 & 0.344712 & 1.001941 & -579.1814 \\
\hline 20 & 0.254328 & -39.49134 & -0.000069 & -459.2803 & 120.6604 & 20.09153 & 0.183067 & 1.000238 & -579.9407 \\
\hline 25 & 0.231031 & -49.53794 & -0.000037 & -390.9432 & 189.2497 & 25.07404 & 0.148084 & 1.000121 & -580.1930 \\
\hline 30 & 0.213366 & -59.57327 & -0.000022 & -307.1928 & 273.2082 & 30.06215 & 0.124291 & 1.000069 & -580.4010 \\
\hline 35 & 0.199350 & -69.60130 & -0.000014 & -208.0429 & 372.5350 & 35.05354 & 0.107070 & 1.000043 & -580.5779 \\
\hline 40 & 0.187864 & -79.62427 & -0.000010 & -93.50292 & 487.2289 & 40.04702 & 0.094034 & 1.000029 & -580.7318 \\
\hline 45 & 0.178218 & -89.64356 & -0.000007 & 36.42054 & 617.2886 & 45.04191 & 0.083823 & 1.000020 & -580.8680 \\
\hline 50 & 0.169963 & -99.66007 & -0.000005 & 181.7226 & 762.7128 & 50.03781 & 0.075611 & 1.000015 & -580.9902 \\
\hline
\end{tabular}

TABLE X: Magnification centroid due to lensing by the Schwarzschild black hole $(\nu=1)$; and weakly $(\nu=0.7)$, marginally strongly $(\nu=0.5)$ and strongly $(\nu=0.04,0.02,0.001)$ naked singularities. (a) The same as (a) of Table I.

\begin{tabular}{|c|c|c|c|c|c|c|}
\hline \multirow[t]{2}{*}{$\beta$} & \multicolumn{6}{|c|}{ Magnification centroid } \\
\hline & $\nu=1(\mathrm{SBH})$ & $\nu=0.7(\mathrm{WNS})$ & $\nu=0.5(\mathrm{MSNS})$ & $\nu=0.04(\mathrm{SNS})$ & $\nu=0.02(\mathrm{SNS})$ & $\nu=0.001(\mathrm{SNS})$ \\
\hline $10^{-6}$ & $1.50000000005 \times 10^{-6}$ & $1.50000000005 \times 10^{-6}$ & $1.50000000004 \times 10^{-6}$ & $1.49999993434 \times 10^{-6}$ & $1.49999895074 \times 10^{-6}$ & $\times$ \\
\hline $10^{-5}$ & 0.0000150000000004 & 0.0000150000000003 & 0.0000150000000003 & 0.0000149999993433 & 0.0000149999895073 & $\times$ \\
\hline $10^{-4}$ & 0.000149999999875 & 0.000149999999875 & 0.000149999999874 & 0.000149999993304 & 0.000149999894944 & $x$ \\
\hline $10^{-3}$ & 0.00149999987032 & 0.00149999987031 & 0.00149999987031 & 0.00149999980461 & 0.00149999882101 & $\times$ \\
\hline $10^{-2}$ & 0.0149998702695 & 0.0149998702694 & 0.0149998702694 & 0.0149998696124 & 0.0149998597771 & $x$ \\
\hline $10^{-1}$ & 0.149870601362 & 0.149870601362 & 0.149870601361 & 0.149870594833 & 0.149870497099 & $\times$ \\
\hline 1 & 1.39699276886 & 1.39699276886 & 1.39699276885 & 1.39699273178 & 1.39699217730 & 1.37347550011 \\
\hline 2 & 2.49070719863 & 2.49070719862 & 2.49070719862 & 2.49070717680 & 2.49070684878 & 2.42816768265 \\
\hline 3 & 3.44974557083 & 3.44974557083 & 3.44974557082 & 3.44974556168 & 3.44974541929 & 3.37969558708 \\
\hline 4 & 4.38823639445 & 4.38823639445 & 4.38823639445 & 4.38823639045 & 4.38823632168 & 4.32856338584 \\
\hline 5 & 5.33392461984 & 5.33392461984 & 5.33392461984 & 5.33392461783 & 5.33392457682 & 5.28599274987 \\
\hline 6 & 6.29011081174 & 6.29011081174 & 6.29011081174 & 6.29011081057 & 6.29011078080 & 6.25179502625 \\
\hline 7 & 7.25521420045 & 7.25521420045 & 7.25521420045 & 7.25521419966 & 7.25521417489 & 7.22426206133 \\
\hline 8 & 8.22719524592 & 8.22719524592 & 8.22719524592 & 8.22719524533 & 8.22719522297 & 8.20182922137 \\
\hline 9 & 9.20438778298 & 9.20438778298 & 9.20438778298 & 9.20438778249 & 9.20438776138 & 9.18329481246 \\
\hline 10 & 10.1855502489 & 10.1855502489 & 10.1855502489 & 10.1855502484 & 10.1855502280 & 10.1677715380 \\
\hline
\end{tabular}

TABLE XI: Magnification centroid shift due to lensing by the Schwarzschild black hole $(\nu=1)$; and weakly $(\nu=0.7)$, marginally strongly $(\nu=0.5)$ and strongly $(\nu=0.04,0.02,0.001)$ naked singularities. (a) The same as (a) of Table I.

\begin{tabular}{|c|c|c|c|c|c|c|}
\hline \multirow[t]{2}{*}{$\beta$} & \multicolumn{6}{|c|}{ Magnification centroid shift } \\
\hline & $\nu=1(\mathrm{SBH})$ & $\nu=0.7(\mathrm{WNS})$ & $\nu=0.5(\mathrm{MSNS})$ & $\nu=0.04(\mathrm{SNS})$ & $\nu=0.02(\mathrm{SNS})$ & $\nu=0.001(\mathrm{SNS})$ \\
\hline $10^{-6}$ & $5.00000000050 \times 10^{-7}$ & $5.00000000047 \times 10^{-7}$ & $5.00000000040 \times 10^{-7}$ & $4.99999934340 \times 10^{-7}$ & $4.99998950741 \times 10^{-7}$ & $\times$ \\
\hline $10^{-5}$ & $5.00000000037 \times 10^{-6}$ & $5.00000000034 \times 10^{-6}$ & $5.00000000028 \times 10^{-6}$ & $4.99999934327 \times 10^{-6}$ & $4.99998950728 \times 10^{-6}$ & $\times$ \\
\hline $10^{-4}$ & 0.0000499999998753 & 0.0000499999998750 & 0.0000499999998743 & 0.0000499999933043 & 0.0000499998949444 & $\times$ \\
\hline $10^{-3}$ & 0.000499999870316 & 0.000499999870313 & 0.000499999870306 & 0.000499999804606 & 0.000499998821007 & $\times$ \\
\hline $10^{-2}$ & 0.00499987026947 & 0.00499987026944 & 0.00499987026938 & 0.00499986961242 & 0.00499985977706 & $\times$ \\
\hline $10^{-1}$ & 0.0498706013618 & 0.0498706013615 & 0.0498706013609 & 0.0498705948326 & 0.0498704970987 & $x$ \\
\hline 1 & 0.396992768862 & 0.396992768859 & 0.396992768855 & 0.396992731780 & 0.396992177305 & 0.373475500109 \\
\hline 2 & 0.490707198627 & 0.490707198624 & 0.490707198620 & 0.490707176798 & 0.490706848782 & 0.428167682651 \\
\hline 3 & 0.449745570828 & 0.449745570827 & 0.449745570824 & 0.449745561683 & 0.449745419290 & 0.379695587076 \\
\hline 4 & 0.388236394450 & 0.388236394449 & 0.388236394447 & 0.388236390447 & 0.388236321681 & 0.328563385839 \\
\hline 5 & 0.333924619843 & 0.333924619842 & 0.333924619841 & 0.333924617833 & 0.333924576816 & 0.285992749873 \\
\hline 6 & 0.290110811742 & 0.290110811742 & 0.290110811741 & 0.290110810567 & 0.290110780796 & 0.251795026246 \\
\hline 7 & 0.255214200451 & 0.255214200451 & 0.255214200450 & 0.255214199661 & 0.255214174885 & 0.224262061328 \\
\hline 8 & 0.227195245922 & 0.227195245922 & 0.227195245921 & 0.227195245327 & 0.227195222966 & 0.201829221370 \\
\hline 9 & 0.204387782975 & 0.204387782975 & 0.204387782975 & 0.204387782486 & 0.204387761378 & 0.183294812456 \\
\hline 10 & 0.185550248860 & 0.185550248859 & 0.185550248859 & 0.185550248432 & 0.185550228011 & 0.167771538043 \\
\hline
\end{tabular}


TABLE XII: Total magnification due to lensing by the Schwarzschild black hole $(\nu=1)$; and weakly $(\nu=0.7)$, marginally strongly $(\nu=0.5)$ and strongly $(\nu=0.04,0.02,0.001)$ naked singularities. (a) The same as (a) of Table I.

\begin{tabular}{|c|c|c|c|c|c|c|}
\hline \multirow[t]{2}{*}{$\beta$} & \multicolumn{6}{|c|}{ Total magnification } \\
\hline & $\nu=1(\mathrm{SBH})$ & $\nu=0.7(\mathrm{WNS})$ & $\nu=0.5(\mathrm{MSNS})$ & $\nu=0.04(\mathrm{SNS})$ & $\nu=0.02(\mathrm{SNS})$ & $\nu=0.001(\mathrm{SNS})$ \\
\hline $10^{-6}$ & $1.38816932538 \times 10^{6}$ & $1.38816932538 \times 10^{6}$ & $1.38816932539 \times 10^{6}$ & $1.38816941645 \times 10^{6}$ & $1.38817077256 \times 10^{6}$ & $\times$ \\
\hline $10^{-5}$ & 138816.932541 & 138816.932541 & 138816.932542 & 138816.941647 & 138817.077259 & $\times$ \\
\hline $10^{-4}$ & 13881.6932808 & 13881.6932809 & 13881.6932809 & 13881.6941915 & 13881.7077527 & $\times$ \\
\hline $10^{-3}$ & 1388.16959552 & 1388.16959552 & 1388.16959553 & 1388.16968659 & 1388.17104270 & $\times$ \\
\hline $10^{-2}$ & 138.819633923 & 138.819633923 & 138.819633924 & 138.819643029 & 138.819778637 & $\times$ \\
\hline $10^{-1}$ & 13.9086926599 & 13.9086926600 & 13.9086926601 & 13.9086935678 & 13.9087070859 & $\times$ \\
\hline 1 & 1.64490824157 & 1.64490824157 & 1.64490824158 & 1.64490830940 & 1.64490931584 & 2.31243076497 \\
\hline 2 & 1.14767800038 & 1.14767800038 & 1.14767800038 & 1.14767801708 & 1.14767826338 & 1.18928813001 \\
\hline 3 & 1.04822623968 & 1.04822623968 & 1.04822623968 & 1.04822624452 & 1.04822631629 & 1.06912991460 \\
\hline 4 & 1.01939069971 & 1.01939069971 & 1.01939069971 & 1.01939070136 & 1.01939072680 & 1.03254298220 \\
\hline 5 & 1.00904161659 & 1.00904161659 & 1.00904161659 & 1.00904161725 & 1.00904162855 & 1.01769251767 \\
\hline 6 & 1.00470884575 & 1.00470884575 & 1.00470884575 & 1.00470884606 & 1.00470885230 & 1.01060207986 \\
\hline 7 & 1.00266919134 & 1.00266919134 & 1.00266919134 & 1.00266919151 & 1.00266919562 & 1.00682076296 \\
\hline 8 & 1.00161696592 & 1.00161696592 & 1.00161696592 & 1.00161696603 & 1.00161696910 & 1.00463247208 \\
\hline 9 & 1.00103306445 & 1.00103306445 & 1.00103306445 & 1.00103306453 & 1.00103306701 & 1.00328346259 \\
\hline 10 & 1.00068928992 & 1.00068928992 & 1.00068928992 & 1.00068928998 & 1.00068929209 & 1.00240885784 \\
\hline
\end{tabular}

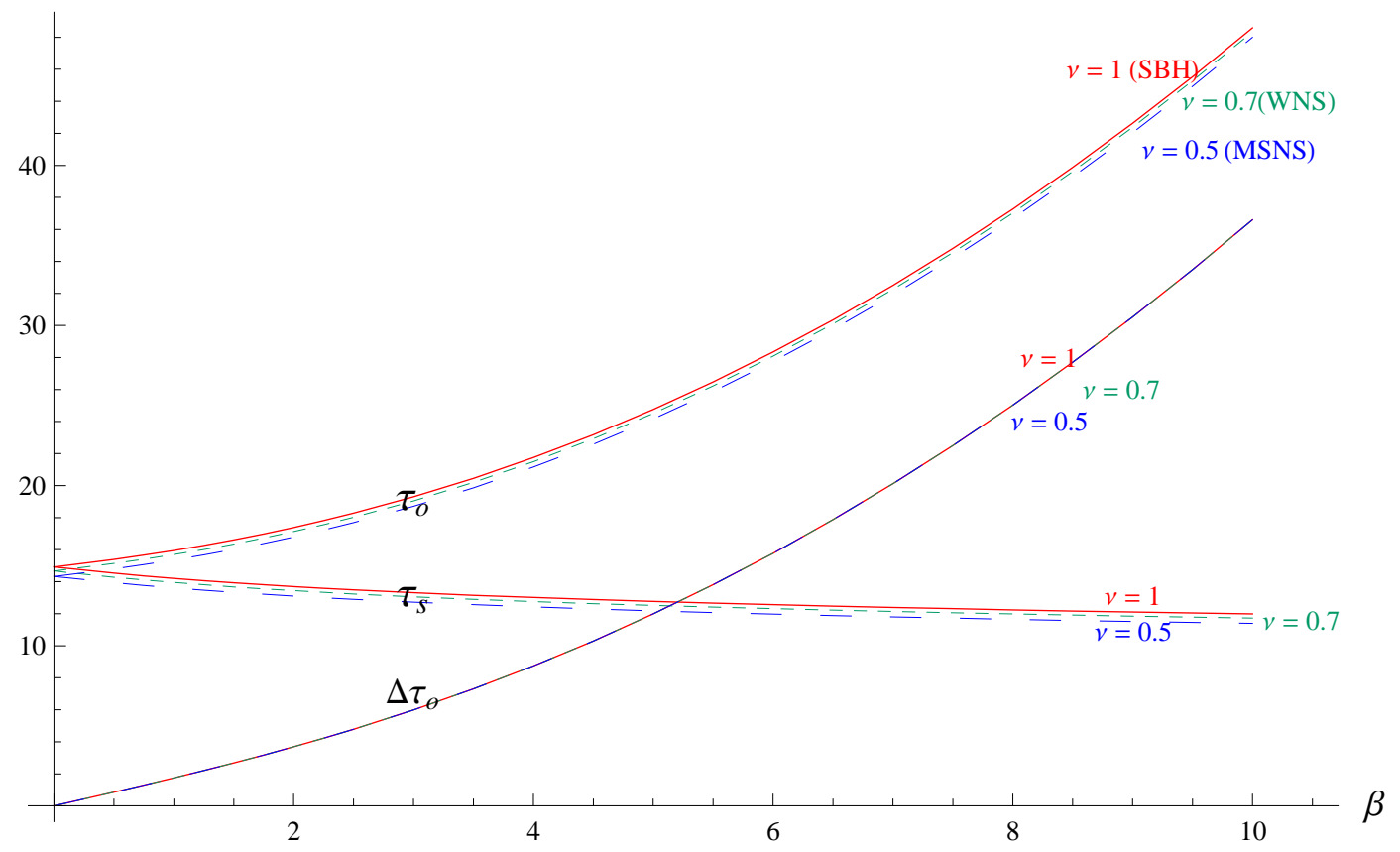

FIG. 1: The time delays of the images on the opposite side from the source $\tau_{o}$, the same side as the source $\tau_{s}$, and the differential time delay of the images on the opposite side from the source $\Delta \tau_{o}$ are plotted against the angular source position $\beta$ for $\nu=1(\mathrm{SBH}), \nu=0.7$ (WNS), and $\nu=0.5(\mathrm{MSNS}) . M / D_{d} \approx 2.26 \times 10^{-11}$ and $D_{d s} / D_{s}=1 / 2$. The time delays as well as differential time delays are expressed in minutes whereas the angular source position is given in arcseconds. 


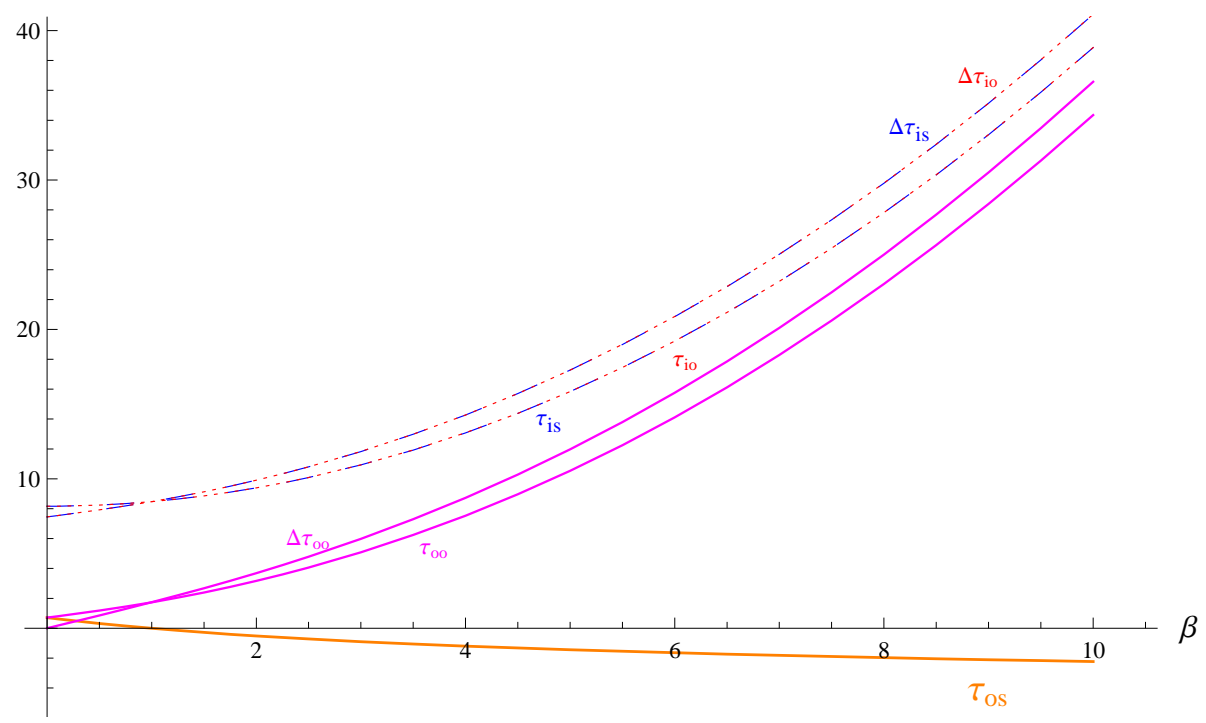

FIG. 2: The time delays of the outer image on the same side as the source $\tau_{o s}$, inner image on the same side as the source $\tau_{i s}$, inner image on the opposite side from the source $\tau_{i o}$, and outer image on the opposite side from the source $\tau_{o o}$ are plotted against the angular source position $\beta$ for $\nu=0.04$ (SNS). Also, the differential time delays of the inner images on the same side as the source $\Delta \tau_{i s}$, inner images on the opposite side from the source $\Delta \tau_{i o}$, and outer images on the opposite side from the source $\Delta \tau_{o o}$ are plotted against $\beta$ for the same value of $\nu . D_{d s} / D_{s}=1 / 2$ and $M / D_{d} \approx 2.26 \times 10^{-11}$. The time delays as well as differential time delays are in minutes whereas the angular source position is expressed in arcseconds.

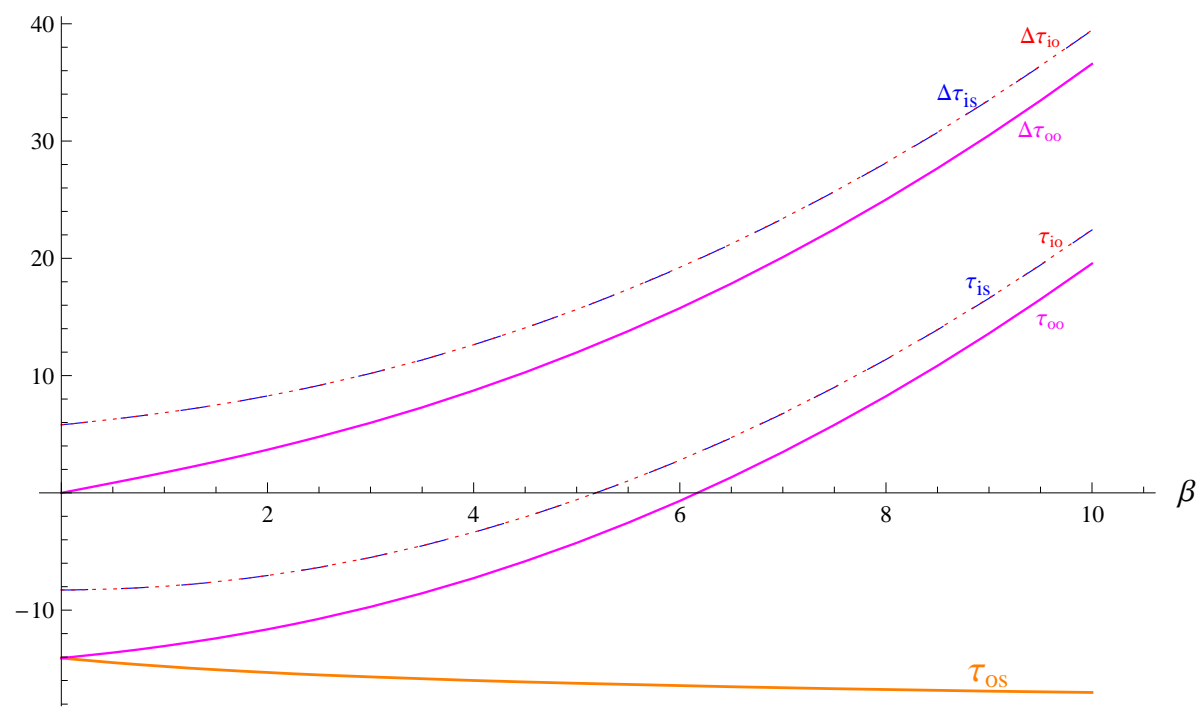

FIG. 3: The time delays of the direct image $\tau_{o s}$, inner image on the same side as the source $\tau_{i s}$, inner image on the opposite side from the source $\tau_{i o}$, and outer image on the opposite side from the source $\tau_{o o}$ are plotted against the angular source position $\beta$ for $\nu=0.02$ (SNS). The differential time delays of the inner images on the same side as the source $\Delta \tau_{i s}$, inner images on the opposite side from the source $\Delta \tau_{i o}$, and outer images on the opposite side from the source $\Delta \tau_{o o}$ are plotted against $\beta$ for the same value of $\nu$. The angular source position is expressed in arcseconds whereas the time delays as well as differential time delays are shown in minutes. $M / D_{d} \approx 2.26 \times 10^{-11}$ and $D_{d s} / D_{s}=1 / 2$.

posite side from the source decreases (the outer and inner images move respectively toward and away from the optical axis) and for some value of $\beta$ these two images coalesce to form one highly magnified image; for example, for $\nu=0.02$, the angular positions of the source and image are $\beta \approx 210.2934$ arcseconds (RC) and $\theta \approx-0.004591$ arcsecond (RCC), respectively. The opposite signs on these two values show that the $\mathrm{RCC}$ and $\mathrm{RC}$ are on the opposite sides of optical axis (see Table I). For further increase in $\beta$, there are no images on the opposite side from the source.

For $\nu=0.001$, see Table IX and Figure 4. If the lens components are aligned, there are no Einstein rings. Also, for small values of $\beta$, there are no images on either 

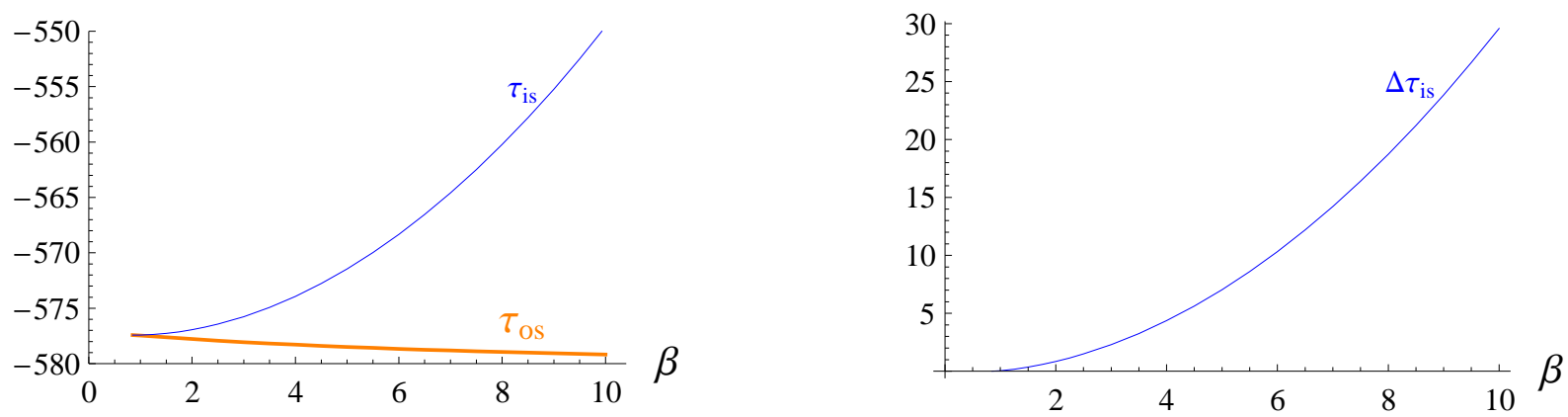

FIG. 4: The time delays of the outer images on the same side as the source $\tau_{\text {os }}$ and inner images on the same side as the source $\tau_{i s}$ are plotted against the angular source position $\beta$ for $\nu=0.001$ (SNS); see the left hand side figure. The differential time delay of the inner image on the same side as the source $\tau_{i s}$ is plotted against $\beta$ (right hand side figure). The time delays as well as differential time delays and the angular source position are respectively given in minutes and arcseconds. $D_{d s} / D_{s}=1 / 2$ and $M / D_{d} \approx 2.26 \times 10^{-11}$.
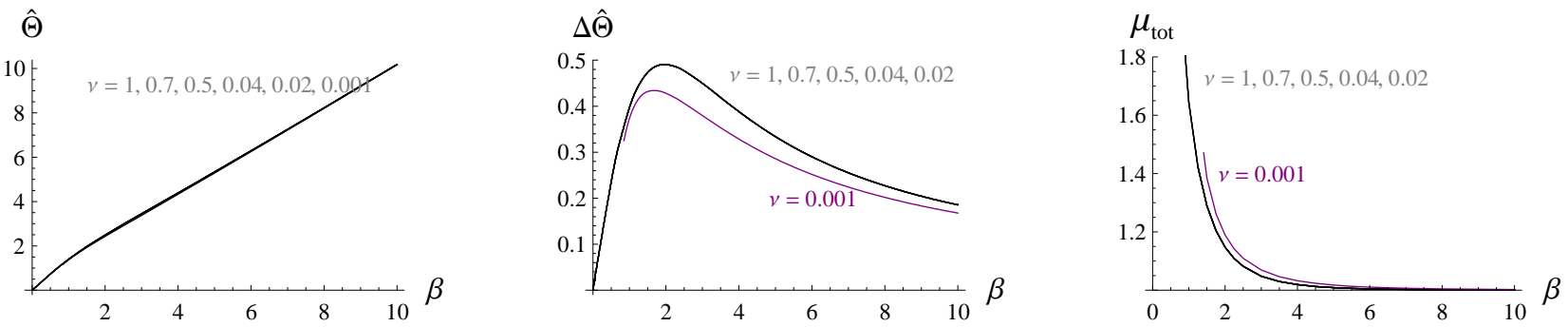

FIG. 5: The magnification centroid $\hat{\Theta}$, magnification centroid shift $\Delta \hat{\Theta}$, and the total magnification $\mu_{\text {tot }}$ are plotted against the angular source position $\beta$ for $\nu=1(\mathrm{SBH}), \nu=0.7$ (WNS), $\nu=0.5(\mathrm{MSNS})$, and $\nu=0.04,0.02,0.001(\mathrm{SNS}) . D_{d s} / D_{s}=1 / 2$ and $M / D_{d} \approx 2.26 \times 10^{-11}$. $\hat{\Theta}, \Delta \hat{\Theta}$, and $\beta$ are expressed in arcseconds.

side of the optical axis. For $\beta \approx 0.808397$ arcsecond $(\mathrm{RC})$, there is a highly magnified image at the angular position $\theta \approx 1.115015$ arcseconds (RCC). The signs on $\mathrm{RC}$ and $\mathrm{RCC}$ being the same implies that this image appear on the same side as the source. As $\beta$ further increases, the image splits into two and the inner and outer (direct) images move toward and away from the optical axis, respectively; the magnification of inner image decreases much faster than the outer one. The time delay of the direct image for this case is always negative and decreases slowly with an increase in $\beta$. The time delay of the inner image, however, is negative for small $\beta$ and positive for large $\beta$, passing through the zero time delay for a certain value of $\beta$. The increase rate of time delay of the inner image is much higher than the decrease rate of time delay of the direct image. For the inner image, the deflection angles are positive and negative respectively for small and large values of $\beta$; however, for the direct image it is always positive. As for other cases already discussed, the differential time delay of the inner image is always positive.

We now compute the magnification centroid, magni- fication centroid shift, and the total magnification for $\nu=1(\mathrm{SBH}), \nu=0.7$ (WNS), $\nu=0.5$ (MSNS), and $\nu=0.04,0.02,0.001$ (SNS) for several values of $\beta$ (see Tables X through XII). We then plot these quantities against $\beta$ (see Fig. 5). For a fixed value of $\nu$, the magnification centroid increases with an increase in $\beta$. For small values of $\beta$, the graph is bulged up and then tends to become straight as $\beta$ increases. For a fixed value of $\beta$, the magnification centroid decreases with a decrease in $\nu$ (i.e., increase in $\left.(q / M)^{2}\right)$; the decrease is however too small for these five graphs to appear resolved even if these were plotted on an entire page. As $\beta$ increases, the magnification centroid shift first increases, reaches a maximum value, and then starts decreasing to the limiting value zero. For a given value of $\beta$, the magnification centroid shift decreases with a decrease in the value of $\nu$. For a given value of $\nu$, the total magnification is, as expected, very high for small $\beta$ and it decreases to the limiting value one, as $\beta$ increases. For any given value of $\beta$, however, the total magnification increases with a decrease in the value of $\nu$. Thus, the presence of scalar charge helps increase the total magnification. This would 
provide a modest increase in the likelihood of observing lensing by $\operatorname{Sgr} A^{*}$ (see [27] and references therein).

All the images produced by the Galactic MDO (modeled as a Schwarzschild black hole and naked singularity lenses) may be resolved from each other by observational facilities available in the near future. Therefore, the results of magnification centroid and total magnification may not be needed. However, our studies help us understand the role of scalar field on the magnification centroid and total magnification, which could be useful while studying gravitational lensing by exotic dark objects having rather small value of $M / D_{d}$.

\section{DISCUSSION AND CONCLUSION}

The naked singularities are classified in three categories: $W N S, M S N S$, and SNS. We modeled the Galactic $\mathrm{MDO}$ as the SBH, and Janis-Newman-Winicour WNS, MSNS and SNS lenses, and studied point source gravitational lensing by them. We found that the gravitational lensing effects due to the SBH, WNS, and MSNS are qualitatively similar (but these differ slightly quantitatively) to each other; however, they differ qualitatively from SNS lensing. Therefore, it will be easier to observationally differentiate a SNS (compared to a WNS or a MSNS) from a SBH.

$S B H, W N S$ and $M S N S$ lensing. These do not give rise to any radial caustics; however, they do produce one Einstein ring when the lens components are aligned (i.e. $\beta=0)$. When $\beta$ increases, the Einstein ring splits into two images, one on each side of the optical axis. The time delays for both images are positive for all values of $\beta$. For a given value of $\beta$, a decrease in $\nu$, i.e., an increase in $(q / M)^{2}$, decreases the absolute angular image positions, time delays, magnification centroid, and magnification centroid shift; however, it increases the magnitude of magnifications of images and therefore also the total magnification. The differences are however very small. The deflection angle $\hat{\alpha}$ becomes unboundedly large as the impact parameter $J \rightarrow J_{p s}$ for the SBH and WNS, and $J \rightarrow 0$ for the MSNS [7].

SNS lensing. There are two types of lensing in this category. In the first, for example for the case of $\nu=0.02$, there are double concentric Einstein rings (when $\beta=0$ ) and one radial critical curve (when $\beta \approx 210.2934$ arcseconds). As the angular position of the source increases from the alignment position of the lens components (i.e., $\beta=0$ ), the two Einstein rings "break" into four images, giving two images on each side of the optical axis. The separation between images on the same and opposite side from the source, respectively, increases and decreases as $\beta$ increases, and eventually the two images on the opposite side from the source coalesce to form a single highly magnified image. For any further increase in $\beta$, there are only two images on the same side as the source. The second category of SNS lensing, for example for $\nu=0.001$, there is one RC; however, there is no Einstein ring when $\beta=0$. Moreover, there is no image for small values of $\beta$. As $\beta$ increases, a highly magnified image (RCC) first appears on the same side as the source. A further increase in $\beta$ splits this into two images and the separation between them keeps increasing (both images remaining on the same side as the source). The time delay of images of SNS lensing may be positive, zero, or negative depending on the values of $\nu$ and $\beta$. However, the time delay of a direct image is negative for any SNS lensing if $\beta$ is large. As shown in [7], the deflection angle $\hat{\alpha}$ approaches $-\pi$ as the impact parameter approaches its minimum value zero. Therefore, if a light ray is sent radially toward a SNS, it will "bounce"; however, it remains to be computed whether or not the "reflected" light has enough magnification to be observed by present instruments or by those likely to be available in near future. This may serve as a crucial test for the existence of SNS.

The naked singularity lensing gives rise to images of smaller time delay and stronger magnification than the black hole lensing. Therefore, if naked singularities indeed exist in nature, then these will serve as better cosmic telescopes and will help probe the universe more efficiently. The results obtained in this paper also helps understand the effects of scalar field on gravitational lensing, which could have valuable implications for research in cosmology.

The Janis-Newman-Winicour metric also describes the exterior gravitational field of a scalar star. Therefore, results obtained in this paper for naked singularities are also applicable to scalar stars. The scalar star however must be compact enough for the images not to be obstructed.

The metric we considered in this paper may or may not be physically realistic. However, gravitational lensing studies with this metric serves as a stepping stone to understand the distinctive lensing features of black holes and naked singularities. It would be indeed of great astrophysical significance to obtain distinguishing qualitative lensing characteristics of Kerr black holes and naked singularities, so that the weak cosmic censorship hypothesis could be tested observationally without any ambiguity.

\section{Acknowledgments}

Thanks are due to J. M. Aguirregabiria of the University of the Basque Country (Spain) for some helpful corresponsence related to Mathematica. 
[1] R. Penrose, in Black Holes and Relativistic Stars, edited by R. M. Wald (The University of Chicago Press, Chicago, 1998), p. 103.

[2] R. M. Wald, "Gravitational Collapse and Cosmic Censorship," gr-qc/9710068

[3] K. S. Virbhadra, Phys. Rev. D 60, 104041 (1999).

[4] K. S. Virbhadra, D. Narasimha, and S. M. Chitre, Astron. Astrophys. 337, 1 (1998).

[5] K. S. Virbhadra and G. F. R. Ellis, Phys. Rev. D 62, 084003 (2000). The light deflection in the vicinity of a photon sphere of a Schwarzschild black hole was also previously studied by some researchers; for example, C. Darwin, Proc. R. Soc. London A 249, 180 (1958); ibid 263, 39 (1961); H. C. Ohanian, Am. J. Phys. 55, 428 (1987); R. J. Nemiroff, ibid, 61, 619 (1993).

[6] C-M. Claudel, K. S. Virbhadra, and G. F. R. Ellis, J. Math. Phys. 42, 818 (2001).

[7] K. S. Virbhadra and G. F. R. Ellis, Phys. Rev. D 65, 103004 (2002).

[8] V. Perlick, Living Rev. Rel. 7, 9 (2004); Commun. Math. Phys. 220, 403 (2001); Phys. Rev. D 69, 064017 (2004); "Theoretical gravitational lensing. Beyond the weak-field small-angle approximation," arXiv:0708.0178 [gr-qc]; W. Hasse and V. Perlick, Gen. Rel. Grav. 34, 415 (2002).

[9] A. F. Zakharov and Yu. V. Baryshev, Class. Quant. Grav. 19, 1361 (2002); Int. J. Mod. Phys. D 11, 1067 (2002); A. F. Zakharov, F. De Paolis, G. Ingrosso and A. A. Nucita, New Astron. 10, 479 (2005).

[10] P. Amore and S. Arceo Diaz, Phys. Rev. D 73, 083004 (2006); P. Amore, S. Arceo and F. M. Fernandez, ibid 74, 083004 (2006); P. Amore, M. Cervantes, A. De Pace and F. M. Fernandez, ibid 75, 083005 (2007).

[11] V. Bozza, S. Capozziello, G. Iovane and G. Scarpetta, Gen. Rel. Grav. 33, 1535 (2001); V. Bozza, Phys. Rev. D 66, 103001 (2002); ibid 67, 103006 (2003); V. Bozza, F. De Luca and G. Scarpetta, ibid 74, 063001 (2006); V. Bozza and L. Mancini, Astrophys. J. 627, 790 (2005).

[12] E. F. Eiroa, G. E. Romero and D. F. Torres, Phys. Rev. D 66, 024010 (2002); E. F. Eiroa and D. F. Torres, ibid 69, 063004 (2004); E. F. Eiroa, Braz. J. Phys. 35, 1113 (2005); Phys. Rev. D 73, 043002 (2006).

[13] T. P. Kling, E. T. Newman and A. Perez, Phys. Rev. D 61, 104007 (2000); ibid 62, 024025 (2000); [Erratumibid. D 62, 109901 (2000)]; S. Frittelli, T. P. Kling and E. T. Newman, ibid 61, 064021 (2000).

[14] A. S. Majumdar and N. Mukherjee, Mod. Phys. Lett. A
20, 2487 (2005); Int. J. Mod. Phys. D 14, 1095 (2005); N. Mukherjee and A. S. Majumdar, Gen. Rel. Grav. 39, 583 (2007).

[15] C. R. Keeton and A. O. Petters, Phys. Rev. D 72, 104006 (2005).

[16] C. R. Keeton and A. O. Petters, Phys. Rev. D , 73, 044024 (2006); ibid, 73, 104032 (2006); A. O. Petters, Mon. Not. Roy. Astron. Soc. 338, 457 (2003); S. V. Iyer and A. O. Petters, Gen. Rel. Grav. 39, 1563 (2007); M. C. Werner and A. O. Petters, Phys. Rev. D 76, 064024 (2007).

[17] A. Bhadra, Phys. Rev. D 67, 103009 (2003); R. Whisker, ibid 71, 064004 (2005); K. K. Nandi, ibid 74,024020 (2006); G. N. Gyulchev and S. S. Yazadjiev, ibid 75, 023006 (2007); M. Safonova and D. F. Torres, Mod. Phys. Lett. A 17, 1685 (2002).

[18] S. Fernando and S. Roberts, Gen. Rel. Grav. 34, 1221 (2002); C. Stornaiolo, Gen. Rel. Grav. 34, 2089 (2002); K. Lake, Phys. Rev. D 65, 087301 (2002); S. E. Vazquez and E. P. Esteban, Nuovo Cim. 119B, 489 (2004); F. Finelli, M. Galaverni and A. Gruppuso, Phys. Rev. D 75, 043003 (2007).

[19] M. P. Dabrowski and F. E. Schunck, Astrophys. J. 535, 316 (2000); T. Matos and R. Becerril, Class. Quant. Grav. 18, 2015 (2001); V. A. De Lorenci, N. Figueiredo, H. H. Fliche and M. Novello, Astron. Astrophys. 369, 690 (2001); F. E. Schunck, B. Fuchs and E. W. Mielke,Mon. Not. Roy. Astron. Soc. 369, 485 (2006); K. Sarkar and A. Bhadra, Class. Quant. Grav. 23, 6101 (2006).

[20] S. Wolfram, MATHEMATICA 6.0.

[21] K. S. Virbhadra, Int. J. Mod. Phys. A 12, 4831 (1997).

[22] K. S. Virbhadra, S. Jhingan, and P. S. Joshi, Int. J. Mod. Phys. D 6, 357 (1997).

[23] P. Schneider, J. Ehlers, and E. E. Falco, Gravitational Lenses (Springer-Verlag, Berlin, 1992).

[24] S. Weinberg, Gravitation and Cosmology: Principles and Applications of the General Theory of Relativity (Wiley, New York, 1972).

[25] A. I. Janis, E. T. Newman, and J. Winicour, Phys. Rev. Lett. 20, 878 (1968); M. Wyman, Phys. Rev. D 24, 839 (1981); M. D. Roberts, Astrophysics \& Space Sc. 200, 331 (1993).

[26] F. Eisenhauer et al., Astrophys. J. 628, 246 (2005).

[27] A. B. Congdon, C. R. Keeton, and C. E. Nordgren, Phys. Rev. D 76 (2007), to appear. 